Document downloaded from:

http://hdl.handle.net/10251/151074

This paper must be cited as:

Leyva-Mayorga, I.; Rodríguez-Hernández, MA.; Pla, V.; Martínez Bauset, J.; Tello-Oquendo, L. (2019). Adaptive access class barring for efficient mMTC. Computer Networks. 149:252264. https://doi.org/10.1016/j.comnet.2018.12.003

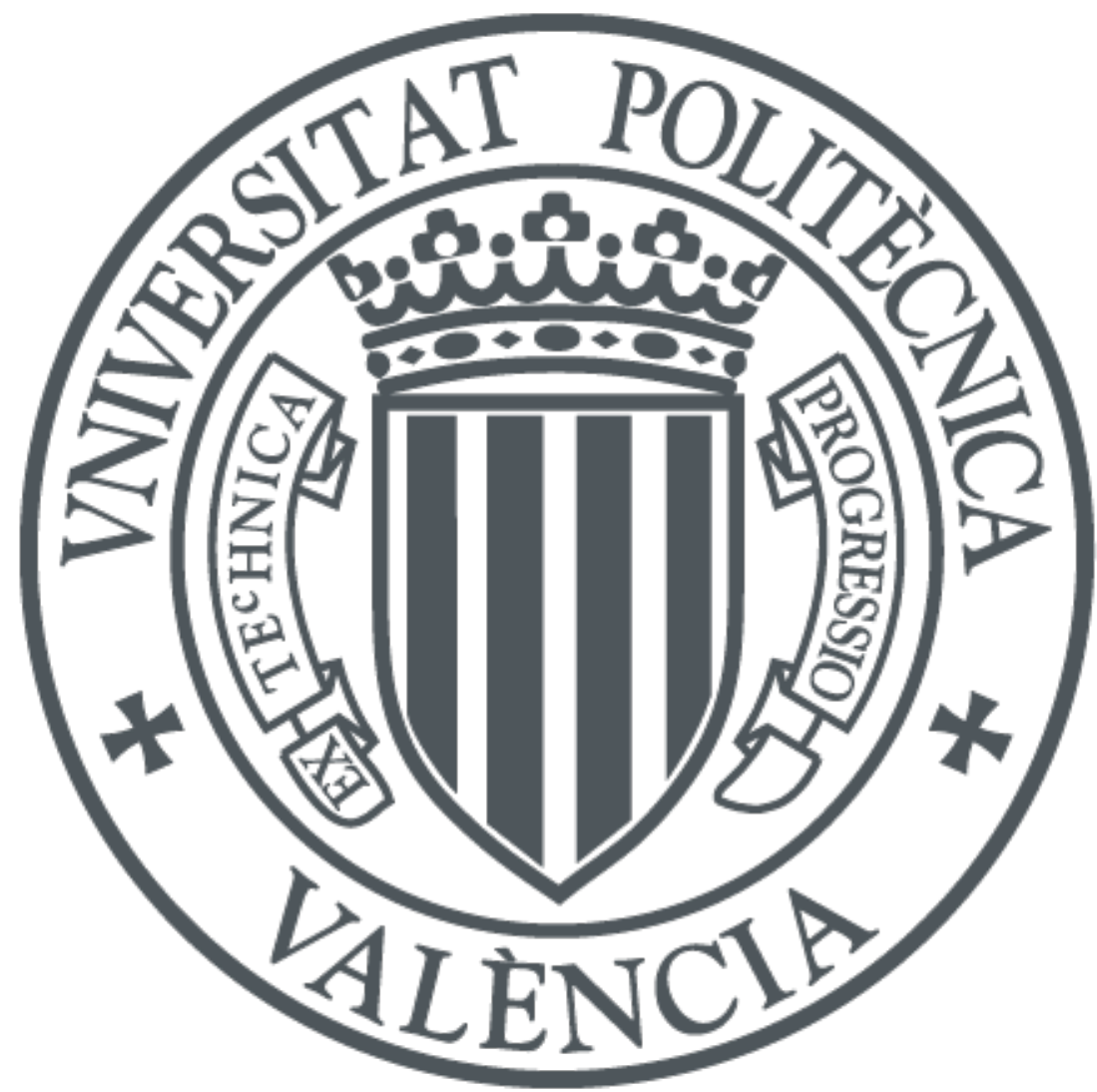

The final publication is available at

https://doi.org/10.1016/j.comnet.2018.12.003

Copyright Elsevier

Additional Information 


\title{
Adaptive access class barring for efficient mMTC
}

Israel Leyva-Mayorga ${ }^{a, *}$, Miguel A. Rodriguez-Hernandez ${ }^{\mathrm{a}}$, Vicent Pla ${ }^{\mathrm{a}}$, Jorge Martinez-Bauset ${ }^{\mathrm{a}}$, Luis Tello-Oquendo ${ }^{\mathrm{b}}$

${ }^{a}$ Instituto ITACA, Universitat Politècnica de València, Camino de Vera, s/n, 46022, Valencia, Spain

${ }^{b}$ College of Engineering, Universidad Nacional de Chimborazo, 060108, Riobamba, Ecuador

\begin{abstract}
In massive machine-type communications (mMTC), an immense number of wireless devices communicate autonomously to provide users with ubiquitous access to information and services. The current $4 \mathrm{G}$ LTE-A cellular system and its Internet of Things (IoT) implementation, the narrowband IoT (NB-IoT), present appealing options for the interconnection of these wireless devices. However, severe congestion may arise whenever a massive number of highly-synchronized access requests occur. Consequently, access control schemes, such as the access class barring (ACB), have become a major research topic. In the latter, the precise selection of the barring parameters in a real-time fashion is needed to maximize performance, but is hindered by numerous characteristics and limitations of the current cellular systems. In this paper, we present a novel ACB configuration (ACBC) scheme that can be directly implemented at the cellular base stations. In our ACBC scheme, we calculate the ratio of idle to total available resources, which then serves as the input to an adaptive filtering algorithm. The main objective of the latter is to enhance the selection of the barring parameters by reducing the effect of the inherent randomness of the system. Results show that our ACBC scheme greatly enhances the performance of the system during periods of high congestion. In addition, the increase in the access delay during periods of light traffic load is minimal.
\end{abstract}

Keywords: Access class barring (ACB); adaptive filters; Internet of Things (IoT); LTE-A; massive machine-type communications (mMTC).

\section{Introduction}

Machine-type communications (MTC) stands for the autonomous exchange of data between devices. This novel paradigm enables a myriad of applications such as smart metering, fleet management, traffic optimization, e-health care, and vehicle control $[1,2]$. As such, achieving efficient MTC is essential to attain a resilient Internet of Things (IoT).

Due to the proliferation of MTC applications, the number of interconnected wireless devices has sharply increased in recent years, and will continue to do so in the near future. For example, the projected number of wireless MTC devices by 2020 is around 3.3 billion [3]. Such a high number of wireless devices poses important challenges on the signaling capabilities of relaying networks. Hence, supporting massive MTC (mMTC) is one of the pillars of $5 \mathrm{G}[1,4]$.

Nowadays, the $4 \mathrm{G}$ LTE-A cellular system is an appealing option for the interconnection of wireless devices (known as user equipments, UEs) in MTC applications because its infrastructure has already been widely deployed,

\footnotetext{
*Corresponding author

Email addresses: isleyma@upv.es (Israel Leyva-Mayorga), marodrig@upv.es (Miguel A. Rodriguez-Hernandez), vpla@upv.es (Vicent Pla), jmartinez@upv.es (Jorge Martinez-Bauset), luis.tello@unach.edu.ec (Luis Tello-Oquendo)
}

and it can meet with high QoS requirements [5]. The current $4 \mathrm{G}$ cellular system will be the base of future $5 \mathrm{G}$ networks. Therefore, significant efforts have been made to efficiently support mMTC in LTE-A. These have led to the development of the narrowband IoT standard (NB-IoT).

NB-IoT is a low-power wide-area (LPWA) implementation in the LTE-A cellular base stations (known as evolved NodeBs, eNBs) that aims to support mMTC by providing great power efficiency, low bandwidth utilization, and enhanced coverage at a reduced hardware cost. For instance, NB-IoT devices are expected to remain active for up to ten years without the need of battery replacements and to communicate at a distance of up to ten kilometers from the eNBs [6]. Nevertheless, the procedure to access the eNB in traditional LTE-A and in NB-IoT is similar.

Specifically, UEs in both LTE-A and NB-IoT shift from idle to connected mode by means of the random access (RA) procedure [7-9]. It is a four-message handshake that comprises preamble transmission (only permitted during random access opportunities, RAOs), random access response (RAR), connection request, and contention resolution messages.

The RA procedure is described in detail in Section 3, still it is important to presently introduce the idea that the first two messages, preamble transmission and RAR, are the main bottlenecks of the RA procedure. Conversely, the RA procedure rarely fails after a RAR has been re- 
ceived because connection request and contention resolution messages are sent through dedicated resources and are protected with robust automatic repeat request (ARQ) mechanisms.

Specifically, the signaling capacity of an eNB is limited by the number of available preambles and by the number of uplink grants that can be sent per RAR message. That is, preambles are orthogonal sequences that are selected randomly by the accessing UEs. Hence, collisions occur when multiple UEs select and transmit the same preamble at the same RAO. On the other hand, uplink grants are sent in response to the successful transmission of an specific preamble and only the UEs that receive an uplink grant can continue with the RA procedure.

Studies have shown that the signaling capacity of an eNB can be easily exceeded when a bulk of UEs transmit in a highly synchronized manner, which is a typical behavior in mMTC applications that leads to severe congestion [1013]. Congestion caused by mMTC applications is a serious problem, as the rapid increase in the number of deployed MTC devices will undoubtedly increase the frequency and severity of congestion in the near future. As a consequence, the development of efficient access control schemes is a hot research topic [14-19].

Among the numerous access control schemes that have been proposed in the literature, the access class barring (ACB) is one of the most promising; hence, it has been included in the LTE-A Radio Resource Control (RRC) specifications [7]. The ACB scheme redistributes the UE access attempts through time. For this, each UE may delay the beginning of its RA procedure according to the barring parameters: barring rate and mean barring time. These parameters are broadcast periodically through the System Information Block Type 2 (SIB2) message. That is, upon arrival, the UEs are allowed to begin the RA procedure (i.e., perform their first preamble transmission at the next RAO) with a probability equal to the barring rate. Otherwise, the beginning of the RA procedure is randomly delayed according to the mean barring time. The ACB scheme is explained in detail in Section 3, yet it is important to emphasize that only the UEs that have not yet performed their first preamble transmission are subject to the ACB scheme [16, 20, 21].

By implementing the ACB scheme, sporadic periods of congestion can be relieved in exchange for a longer access delay if the barring parameters are correctly configured. This is true even if barring parameters remain fixed throughout the congestion period $[13,16,18,22]$. However, congestion is a transitory phenomenon, so an optimal performance can only be obtained by continuously adapting the barring parameters to the traffic intensity. Doing otherwise would increase the access delay of UEs during intervals of low signaling traffic intensity (i.e., under normal operating conditions where no congestion occurs) and fail to relieve congestion episodes that are more severe than expected.

Numerous methods to continuously adapt the barring parameters have been proposed in the literature $[17,23-$ 25]. Throughout this paper we refer to these methods simply as ACB configuration (ACBC) schemes. In theory, some of these schemes can achieve a near-optimal performance. That is, maximize the utilization of resources and guarantee the access of the vast majority of the UEs to the eNB. However, up to now, such a high performance has only been obtained by assuming an idealized ACB scheme and by setting extremely precise barring parameters, for which complex processes and bold assumptions are oftentimes needed. As a consequence, these ACBC schemes cannot be implemented in the current cellular systems.

In the practice, the development of an ACBC scheme capable of adapting its parameters in a real-time fashion is a challenging task [25] that is mainly hindered by:

1. The limited information available at the eNB regarding the number of contending UEs: The eNB ignores the number of contending UEs at a given time, the exact number of UEs deployed within the cell and, clearly, the distribution that the UE accesses will follow before these occur. After each RAO, the eNB is clearly aware of the number of successful accesses, but the number of failed accesses may not be known as several causes for an access failure exist. Please refer to [16] for more details on this matter. Therefore, the eNB can only approximate the number of UEs deployed in a cell based on the number of UEs registered previously and the number of successful accesses at each RAO. Needless to say, the accuracy of such an approximation will suffer.

2. The delay of notification mechanisms: The barring parameters are broadcast through the SIB2, whose period is much longer than the period of RAOs. Therefore, it is not possible to set precise barring parameters in a RAO-by-RAO basis.

3. The selectivity of the ACB scheme: Only the UEs that have not yet begun the RA procedure are subject to the ACB scheme. That is, those who have not yet performed their first preamble transmission. Once a UE has transmitted its first preamble, it is no longer subject to the ACB scheme [7, 21].

In this paper, we present an adaptive ACBC scheme that can be directly implemented in current cellular systems. Our ACBC scheme relies on the number of UEs that successfully complete the RA procedure and in an adaptive filtering process to adjust the barring parameters to the perceived signaling traffic intensity. The main objective of the filtering process is to enhance the selection of the barring rate by reducing the effect of the inherent randomness of the distribution of UE accesses and of the RA procedure. The contributions of our ACBC scheme are as follows.

1. It effectively operates with minimal information regarding the signaling traffic intensity. In fact, only 
the number of successful accesses per RAO and the total amount of available resources are needed to accurately set the barring parameters. These are clearly known by the eNB.

2. It efficiently tolerates the long periodicity of the SIB2. As mentioned above, the barring parameters are exclusively broadcast through the SIB2, whose shortest period is $80 \mathrm{~ms}$, as defined in the specifications [7]. In a typical configuration of the Physical Random Access Channel (PRACH), RAOs occur once every $5 \mathrm{~ms}$. Therefore, the period of the SIB2 is typically 16 times longer than the period of RAOs [7].

3. It successfully configures the barring parameters of the ACB scheme as defined in the RRC specification [7]. Hence, it efficiently relieves congestion given that the ACB scheme only affects the UEs that have not yet begun the RA procedure.

Our ACBC scheme incorporates the simple and robust least-mean-square (LMS) algorithm to continuously adapt the filter weights. Concretely, two different configurations of the LMS are considered. The first one is a typical adaptive line enhancer (ALE) configuration, whose purpose is to remove a wideband noise from a narrowband information-bearing signal. The second one is a novel twist on the typical ALE configuration. Preliminary results on the performance analysis of our ACBC scheme with this second configuration can be found in [26].

We assess the performance of our ACBC scheme by means of the idealized scheme with full state information presented by Duan et al. [17]; it was also used for benchmark purposes in their work. This scheme cannot be implemented in LTE-A but serves as an upper bound to the performance of ACBC schemes. Results show that a remarkable performance can be obtained by implementing our ACBC scheme with either of the two adaptive filter configurations. For instance, the probability of successfully completing the RA procedure during periods of congestion can go from a poor 31.3 percent with no implemented ACB scheme to more than 95 percent with our ACBC scheme. In addition, the difference in the access delay of UEs when compared to the benchmark scheme under these conditions is minor. Moreover, the access delay is not affected during periods of no congestion. These characteristics make our ACBC scheme an efficient solution to the congestion caused by mMTC applications.

The rest of the paper is organized as follows. The state of the art on ACBC schemes is presented in Section 2. The ACB scheme and the RA procedure of LTE-A as defined in the protocol specifications are described in detail in Section 3. Next, our novel ACBC scheme is presented in Section 4, where the employed adaptive algorithms and configurations are also thoroughly described. The scenarios, the benchmark scheme, and the methodology for the performance evaluation of our ACBC scheme are characterized in Section 5. Relevant numerical results are pre- sented and discussed in Section 6. Finally, conclusions are presented in Section 7.

\section{Related work}

One of the most promising and widespread approaches to implement an ACBC scheme is to estimate the total number of contending UEs. With such an approach, an optimal barring rate can be selected. The optimal barring rate is typically defined as the barring rate that maximizes the expected number of preambles transmitted by exactly one UE per RAO [27]. For instance, a closed-form approximate solution to the problem of obtaining the optimal barring rate is presented in [27].

Following this line, a pseudo-Bayesian ACBC scheme is proposed in [28]. In this scheme, the number of idle preambles is used to estimate the number of active UEs and, hence, select an optimal barring rate at each RAO. The use of a Kalman filter is proposed in [23] to enhance the accuracy of the estimation of the number of contending UEs at each RAO. While this study only considers the first step of the RA procedure, preamble transmission, the idea of using adaptive filters for this purpose is promising.

Duan et al. presented an ACBC scheme [17] in which the number of successful and idle preambles (i.e., not transmitted by any UE), along with the total number of previously registered UEs, are used to estimate the number of contending UEs. With this information, the optimal barring rate at each RAO is calculated. An extension of this latter scheme is also provided to dynamically select the number of available preambles allocated to MTC devices.

Abbas et al. [19] present an ACBC scheme that resembles the ACBC scheme with fixed preambles presented by Duan et al. [17] and additionally incorporates the assignment of different barring rates for different groups of UEs based on their delay requirements. As we will observe in Section 6, the access delay of UEs under any ACB scheme and during periods of congestion is only suitable for delay tolerant applications, even when the optimal barring parameters are selected. Hence, the potential delay gains of using different barring parameters for different groups of UEs are minimal.

The performance of the above mentioned ACBC schemes is typically compared with that of idealized solutions that exploit the benefits of having full state information and can make a priori decisions [17, 23, 27]. Clearly, these full state information solutions cannot be implemented but provide an upper bound to the performance of the ACB scheme. In this paper we also compare the performance of our ACBC scheme with that of the idealized and full state information scheme presented by Duan et al. [17].

Results presented in $[17,23,27]$ show that the performance of the proposed ACBC schemes is close to that of the idealized solution. Nevertheless, these ACBC schemes 
cannot be implemented in current cellular networks because were developed based on an idealized ACB scheme in which: 1) every UE is subject to the ACB scheme, even after the beginning of the RA procedure and 2) the barring rate is calculated and broadcast by the eNB at each RAO.

Yet another factor that hinders the implementation of the ACBC schemes mentioned above is that, in order to accurately approximate the number of contending UEs, the eNB must be aware of the number of preambles transmitted by exactly one UE and also of at least one of the following: 1) the number of preambles not transmitted by any UE (i.e., idle preambles); or 2) the number of preambles transmitted by more than one UE (i.e., collisions). In a real world implementation, this information may not be available and the reasons for this are manifold. The interested reader is referred to studies such as [11, 16, 25] for more information on this matter.

De Andrade et al. [25] proposed and evaluated the performance of an ACBC scheme, along with several other access control schemes. The presented schemes consider the delay of each notification mechanism and their performance is assessed in terms of numerous KPIs. Results show that their ACBC scheme leads to the highest success probability (i.e., the probability to successfully complete the RA procedure) under a highly congested scenario. Nevertheless, the obtained success probability is lower than 0.8 .

Finally, Tello-Oquendo et al. [29] presented an ACBC scheme that incorporates a reinforcement learning technique. The proposed ACBC scheme may indeed be implemented in cellular networks as it was designed with the restrictions described above. For instance, the shortest period of the SIB2 was considered. On the other hand, the results obtained with this ACBC scheme were not entirely satisfactory. That is, a sufficiently high success probability can be obtained with this ACBC scheme under a highly congested scenario, but the access delay is more than 25 percent longer when compared to a near-optimal implementation of the ACB with fixed parameters.

Results presented by de Andrade et al. [25] and by Tello-Oquendo et al. [29] showcase the difficulty of designing ACBC schemes and the impact that the delay of notification mechanisms have on performance. As will be shown in Section 4, our ACBC scheme can be directly implemented in cellular networks as it considers each and every one of the system limitations and can lead to a success probability higher than 95 percent.

\section{Random access in LTE-A}

In this section, we describe the $\mathrm{ACB}$ scheme and the contention-based RA procedure as defined in the specifications [7-9]. A brief description of these is provided in Fig. 1.

In order to switch from idle to connected mode, the UEs first acquire the network configuration parameters;

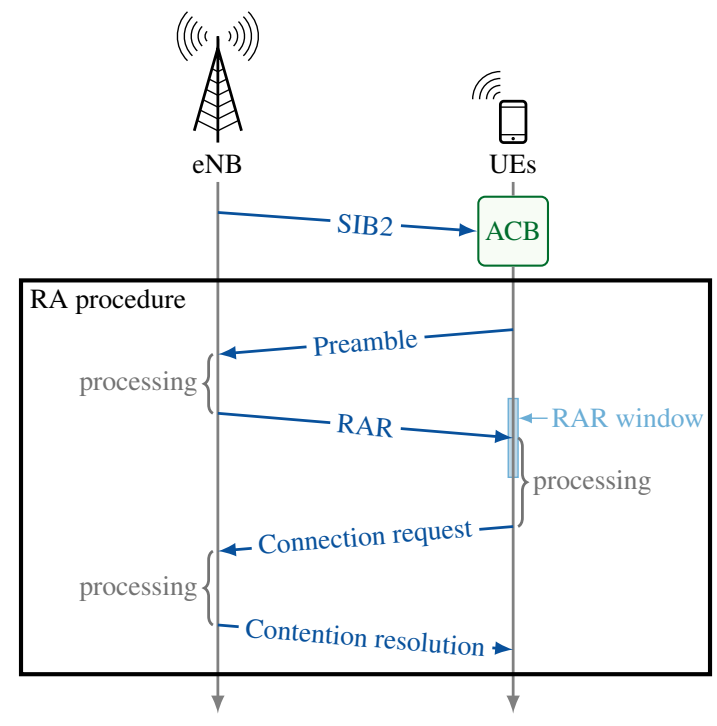

Figure 1: Random access (RA) in LTE-A: ACB scheme and RA procedure

then, they continue to the ACB scheme and, finally, the RA procedure. The network operates in a time-slotted channel in which the minimum unit for scheduling is the subframe.

The configuration parameters are broadcast by the eNB through the System Information Blocks (SIBs). The SIB1 includes, among other parameters, the periodicity of other SIBs in the parameter si-Periodicity $\epsilon$ $\{80,160, \ldots, 5120\} \mathrm{ms}[7]$. The SIB2 includes some basic parameters such as the periodicity of the time/frequency resources in which preamble transmissions are allowed $t_{\text {rao }}$ (known as random access opportunities, RAOs). The SIB2 also includes the barring parameters [7, 9, 12, 13].

Upon arrival, the UEs are subject to the ACB scheme and are divided into access classes (ACs) 0 to 15 according to their traffic characteristics. Each UE belongs to one out of the ten normal ACs (from ACs 0 to 9), and can also belong to one or more out of the high-priority categories (AC 10 is for emergency calls and ACs 11 to 15 are special ACs). The $j$ th SIB2 transmission includes a barring rate $p_{\text {acb }}(j)$ and a mean barring time $t_{\mathrm{acb}}(j)$ that are applied to all ACs 0 to 9 , and to one or more of the ACs 10 to 15. The list of the high-priority categories that are subject to the ACB scheme is also included in the SIB2 [7].

UEs subject to the ACB scheme must perform a barring check before initiating the RA procedure (i.e., before the transmission of their first preamble) as described in Algorithm 1 [7, 8].

UEs that succeed in the barring check are no longer subject to the ACB scheme [21] and proceed to perform the RA procedure as follows.

Preamble (Msg1): Each UE randomly selects one out of the $r$ available preambles and sends it towards the eNB in a RAO through the random access channel (RACH). Preambles in LTE-A are orthogonal (i.e., Zadoff- 


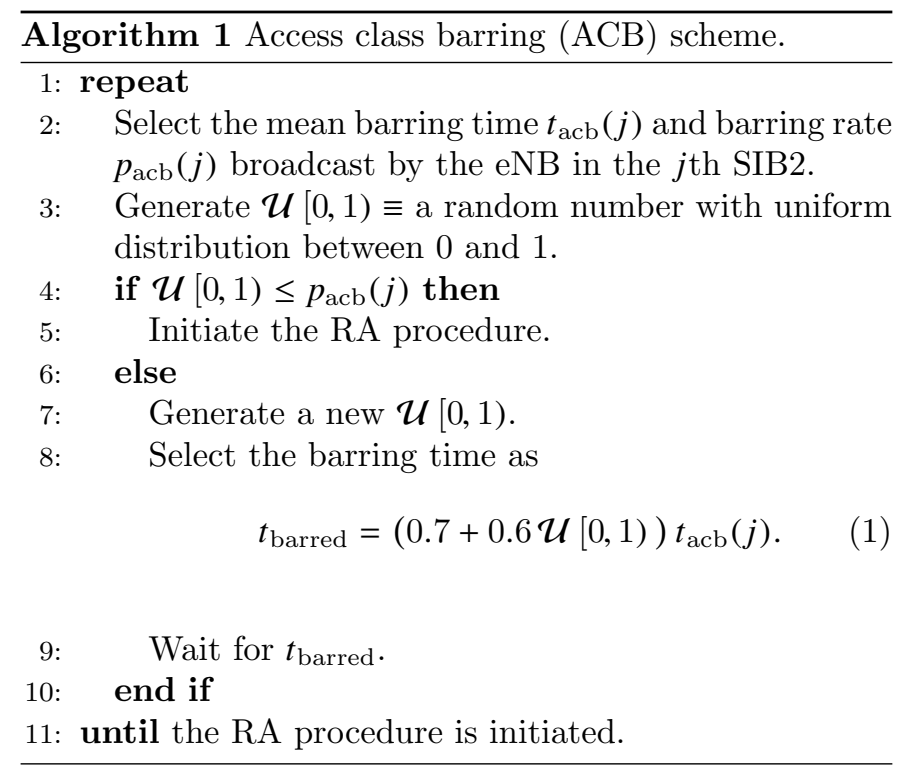

$\mathrm{Chu}$ ) sequences, whereas in NB-IoT these are orthogonal single-tone frequency-hopping patterns. Therefore, multiple UEs can access the eNB in the same RAO using different preambles. The eNB decodes the preambles transmitted with sufficient power by exactly one UE.

On the other hand, two possible outcomes exist when the same preamble is transmitted by multiple UEs. In the first one, a collision occurs and the eNB does not decode the transmitted preamble. In the second one, the eNB correctly decodes the transmitted preamble. In this study we assume that the eNB only decodes preambles transmitted by exactly one UE; hereafter we refer to these as successful preambles. This assumption goes in line with the $3 \mathrm{GPP}$ recommendations for the performance analysis of the RACH [10] and with most of the literature [11, 22$24,30-32]$. The interested reader may refer to [16] for more details on the multiple causes for these two different outcomes, along with the two main assumptions related to the RA procedure and their impact on performance.

Processing the preambles at the eNB takes two subframes. After this period, the UE begins to wait for a RA response (RAR) window of length $w_{\text {rar }}$ subframes to receive a RAR message from the eNB.

RAR (Msg2): The eNB computes an identifier for each successfully decoded preamble and sends the RAR message through the physical downlink control channel (PDCCH). It includes, among other data, uplink grants for the transmission of Msg3. There can be up to one RAR message in each subframe, but it may contain several uplink grants; each of which is associated to a successfully decoded preamble.

The PDCCH resources are limited, so a maximum of $n_{\text {ug }}$ uplink grants can be sent within a RAR window. Specifically, up to three uplink grants can be sent per subframe in a RAR message; hence, the number of available uplink grants per RAR window is given as $n_{\mathrm{ug}}=3 w_{\mathrm{rar}}$.

Connection request ( $\boldsymbol{M s g}$ 3): After receiving the corresponding uplink grant, the UEs adjust their uplink transmission time according to the received time alignment and schedule the transmission of the connection request message, $M s g 3$, to the eNB through dedicated resources.

Contention resolution ( $\boldsymbol{M s g} 4$ ): The eNB transmits a contention resolution message in response to each received $M s g 3$. If a UE does not receive $M_{s g} 4$ within a predefined time window known as the Contention Resolution Timer, then it declares a failure in the contention resolution and schedules a new preamble transmission.

The maximum number of allowed preamble transmissions for each UE is broadcast by the eNB through the SIB2 [7]. Whenever an access attempt fails (e.g., due to a preamble collision) and, if the maximum number of preamble transmissions has not been reached, the UE waits for a random backoff time (determined by the backoff indicator); then randomly selects and transmits a new preamble (Msg1) at the next RAO.

The RA procedure in NB-IoT is greatly similar to that in LTE-A with a few minor exceptions [33]. Concretely, the single difference between NB-IoT and LTE-A that has an impact on performance is that up to three coverage enhancement (CE) levels can be defined in NB-IoT (CE levels zero, one, and two). The CE level of a given UE defines the number of preamble repetitions to be performed one after another per each access attempt. Concretely, only one repetition performed at $\mathrm{CE}$ level zero, and the number of preamble repetitions increases with the CE level. Preamble repetitions are meant to reduce the probability of an access failure due to wireless channel errors [34, 35]. It is also important to mention that the preambles assigned to each CE may be different.

Every UE belongs to CE level zero unless the quality of the measured reference signals sent by the eNB is poor due to an unfavorable wireless environment, or the UE has reached the maximum number of access attempts successfully. In the latter case the UE increases its CE level.

As such, the number of UEs in CE levels one and two is expected to be a relatively low number when no congestion has occurred. Building on this, in this study we focus on the access control of the UEs in CE level zero as these contribute the most to congestion in the PRACH and assume that different preambles have been assigned to each CE level.

\section{Access class barring configuration (ACBC) scheme}

In this section we describe in detail the operation of our novel ACBC scheme. It is important to emphasize that a remarkable feature of our $\mathrm{ACBC}$ scheme is that it strictly adheres to the ACB scheme as defined in the specifications $[7,8]$. That is, we provide an efficient method to calculate adequate parameters for the ACB scheme defined in 


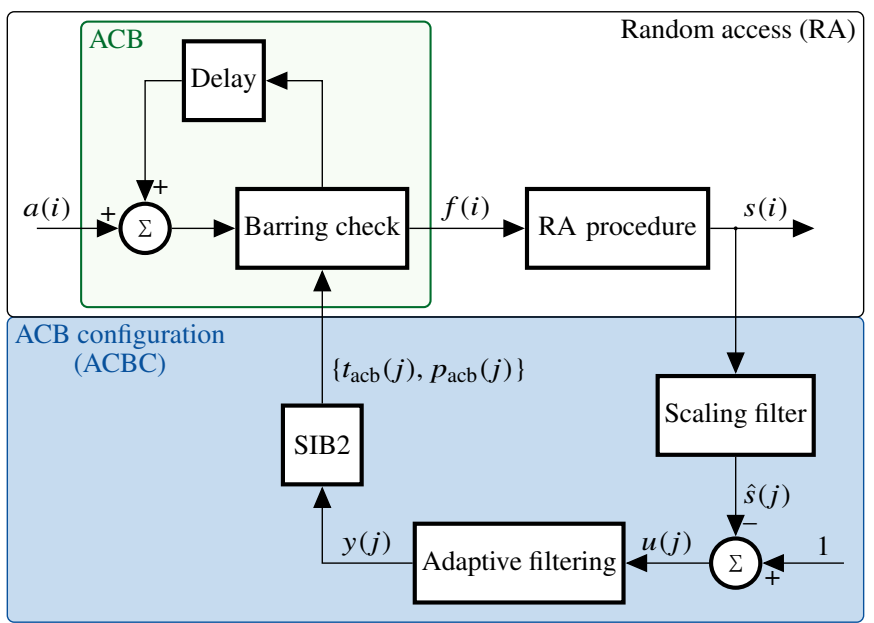

Figure 2: Block diagram of the LTE-A RA procedure with our novel ACBC scheme. The random access is performed at each RAO, whereas the $\mathrm{ACBC}$ can only be performed once every $t_{\mathrm{si}}$ RAOs.

the standards. Therefore, it can be directly implemented at the eNBs.

The block diagram that describes the operation of the RA procedure with our ACBC scheme is shown in Fig. 2, from which two main blocks are clearly identified: random access (RA) and ACB configuration (ACBC). The RA, depicted in the upper part of Fig. 2, operates as described in Section 3.

Let $a(i)$ be the number of UEs that attempt to switch from idle to connected mode for the first time at the $i$ th RAO (i.e., UE arrivals). These UEs are subject to the ACB scheme.

Next, let $f(i)$ be the number of UEs whose barring check at the $i$ th RAO is successful. These UEs proceed to perform the RA procedure; hence, $f(i)$ is also the number of UEs whose first preamble is transmitted at the $i$ th RAO. Finally, $s(i)$ is the number of UEs that have successfully transmitted a preamble at the $i$ th RAO and that will receive an uplink grant within the $i$ th (i.e. next) RAR window. Clearly, the RA is performed at each and every RAO. Therefore, the discrete time index $i$ stands for the epoch number, where the epoch duration is one RAO.

In the ACBC block, depicted in the lower part of Fig. 2, the eNB calculates the barring parameters: mean barring time $t_{\mathrm{acb}}(j)$ and barring rate $p_{\mathrm{acb}}(j)$ that will be broadcast through the $j$ th SIB2. The SIB2 is broadcast once every $t_{\mathrm{si}}$ RAOs, hence, these parameters are adapted according to the perceived signaling traffic intensity throughout this period. As such, the discrete time index $j$ stands for the epoch number when the epoch duration is $t_{\mathrm{si}}$ RAOs. Consequently, the ACBC block operates at a time scale that is $t_{\mathrm{si}}$ times greater than that of the RA block. Specifically, the $j$ th SIB2 is broadcast at the $i=\left(j t_{\mathrm{si}}\right)$ th RAO. Therefore, $p_{\mathrm{acb}}(j)$ remains constant from the $\left(j t_{\mathrm{si}}+1\right)$ th until the $\left((j+1) t_{\mathrm{si}}\right)$ th RAO. We now describe in detail the processes to calculate the barring parameters $p_{\text {acb }}(j)$ and $t_{\text {acb }}(j)$.
The eNB calculates the ratio of utilized to available resources immediately before the $j$ th SIB2 transmission. For this, let $R_{s}$ be the RV that defines the number of successful preambles at the a given RAO. Also let $n(i)$ be the number of contending UEs (i.e., total number of preamble transmissions) at the $i$ th RAO. From there, the theoretical PRACH (uplink) capacity can be defined as the maximum expected value of $R_{s}$ for a given number of available preambles $r$ and for any $n(i) \in \mathbb{R}_{\geq 0}$. The PRACH capacity is achieved when the number of contending UEs is $n(i)=[\log (r /(r-1))]^{-1}$ and is calculated in [24] as follows.

$$
c(r)=\left[\log \left(\frac{r}{r-1}\right)\right]^{-1}\left(1-\frac{1}{r}\right)^{\left[\log \left(\frac{r}{r-1}\right)\right]^{-1}-1} .
$$

In previous studies [20], we found that

$$
c(r) \approx r\left(1-\frac{1}{r}\right)^{r-1} \approx \frac{r}{e},
$$

where $e$ is the Euler's number.

Next, let $c\left(r, n_{\mathrm{ug}}\right)$ be the theoretical capacity of the channels involved in the RA procedure: $\mathrm{PRACH}$ and PD$\mathrm{CCH}$. The capacity of the PDCCH is simply determined by the number of available uplink grants per RAR window, $n_{\text {ug }}[10]$. Hence, we have

$$
c\left(r, n_{\mathrm{ug}}\right)=\min \left\{c(r), n_{\mathrm{ug}}\right\} .
$$

That is, the capacity of the RA procedure is the minimum between the PRACH capacity and the PDCCH capacity. We refer to $c\left(r, n_{\mathrm{ug}}\right)$ simply as the signaling capacity.

With this information, the ratio of utilized to available resources is calculated in the scaling filter block shown in Fig. 2 immediately before the $j$ th SIB2 transmission (i.e., at the $\left(j t_{\mathrm{si}}\right)$ th $\left.\mathrm{RAO}\right)$ as

$$
\hat{s}(j)=\frac{1}{t_{\mathrm{si}} c\left(r, n_{\mathrm{ug}}\right)} \sum_{i=(j-1) t_{\mathrm{si}}+1}^{j t_{\mathrm{si}}} s(i) .
$$

Then, let $u(j)$ be the ratio of idle to available resources for the $j$ th SIB2 broadcast interval. It is easily calculated as

$$
u(j)=1-\hat{s}(j) .
$$

Next, $u(j)$ serves as the input to the adaptive filtering process, whose output $y(j)$ is used to calculate the barring rate for the $j$ th SIB2 broadcast interval

$$
p_{\text {acb }}(j)=\min \{y(j), 1\} .
$$

Hence, $p_{\text {acb }}(j)$ decreases with the ratio of idle to available resources. This increases the probability of delaying the beginning of the RA procedure when most of the resources have been utilized.

Finally, we propose the dynamic selection of the mean barring time $t_{\mathrm{acb}}(j)$ as a function of $p_{\mathrm{acb}}(j)$. For this, let $t_{\max }$ be the longest mean barring time that can be broadcast by the eNB. Hereafter we refer to $t_{\max }$ simply as 
the barring indicator; it is fixed and selected empirically by the network administrator. Then, the mean barring time is calculated as

$$
t_{\mathrm{acb}}(j)=\left(1-p_{\mathrm{acb}}(j)\right)^{\omega} t_{\max } ;
$$

where exponent $\omega \in \mathbb{R}_{\geq 0}$. The impact of parameter $\omega$ on the performance of our ACBC scheme is discussed in Section 6 . We now proceed to describe the selected adaptive algorithm and the two different configurations that were implemented in our ACBC scheme.

\subsection{Adaptive filtering algorithm configurations}

Our ACBC scheme incorporates an adaptive filtering algorithm to continuously adapt the weights of a filter according to the ratio of idle to available resources. While any adaptive algorithm can be incorporated, we have selected the simple and robust least-mean-square (LMS) algorithm. The latter is widely used because of its simplicity and numerical robustness [36]. Concretely, the complexity of the LMS algorithm is $O(M)$, where $M$ is the filter length. That is, it scales linearly with the filter length as $2 M+1$ multiplications and $2 M+1$ additions are performed per iteration (i.e., per ACBC process) [36]. Since the eNBs possess great computational power, they can easily implement the LMS algorithm. A widely used alternative to the LMS is the recursive-least squares (RLS) algorithm, but our initial analyses revealed that the benefits of the LMS outperform those of the RLS for the target application.

The block diagram of the LMS adaptive algorithm is shown in Fig. 3. A buffer has been incorporated to clearly illustrate that the ratio of idle resources during the last $M$ SIB2 intervals,

$$
\boldsymbol{u}(j)=[u(j), u(j-1), \ldots, u(j-M+1)],
$$

serves as the input to the algorithm. In other words, a single value of $u(j)$ is the input to the buffer as indicated by the thin arrow, and the output of the buffer is a vector as indicated by the thick arrows.

As Fig. 3 shows, the LMS algorithm consists of two processes: the filtering and the adaptive process, which result in a feedback loop. In the filtering process, the output of a finite-duration impulse response (FIR) filter $y(j)$ is computed from $\boldsymbol{u}(j)$.

In the adaptive process, the output $y(j)$ is compared to the desired response $d(j)$ to obtain the a priori error $e(j)$. Then, $e(j)$ serves as an input to the adaptive weight control mechanism. The latter is in charge of adapting the weights of the FIR filter

$$
\boldsymbol{w}(j)=\left[w_{0}(j), w_{1}(j), \ldots, w_{M-1}(j)\right],
$$

automatically based on $e(j)$ and on the adaptation step size $\mu$. The LMS adaptive algorithm is summarized in Algorithm 2.

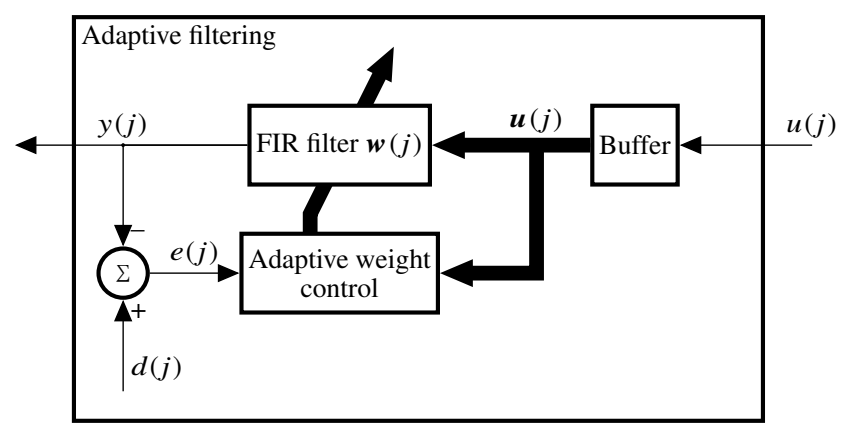

Figure 3: Block diagram of the LMS adaptive algorithm.

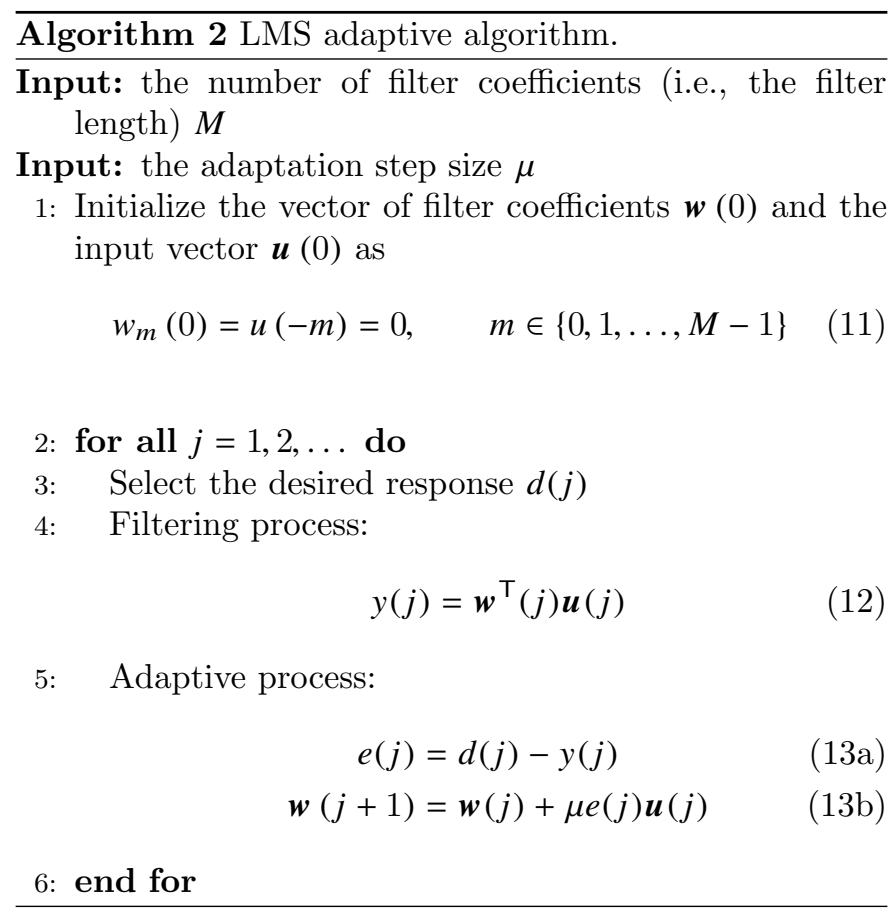

It is important to mention that $\mu$ determines the socalled energy constraint or rate of adjustment $\alpha$, which links the a priori error $e(j)$ with the a posteriori error $\varepsilon(j)$ as follows [37, Chapter 5.3].

$$
\begin{aligned}
\varepsilon(j) & =d(j)-\boldsymbol{u}^{\top}(j) \boldsymbol{w}(j+1) \\
& =d(j)-\boldsymbol{u}^{\top}(j)(\boldsymbol{w}(j)+\mu \boldsymbol{u}(j) e(j)) \\
& =(1-\alpha(j)) e(j)
\end{aligned}
$$

where $\alpha(j)=\mu\|\boldsymbol{u}(j)\|^{2}$ is the energy constraint at time index $j$ and $\|\cdot\|$ is the Euclidean norm operator. As such, parameter $\alpha$ determines the rate at which $\boldsymbol{w}(j)$ is adjusted, based on $\boldsymbol{u}(j)$.

For the LMS algorithm to be stable, the value of $\mu$ must satisfy [36-38]

$$
|1-\alpha(j)| \leq 1 \quad \forall j
$$

which gives

$$
0<\alpha_{\max } \leq 2
$$


where $\alpha_{\max }=\mu \max \left\{\|\boldsymbol{u}(j)\|^{2}\right\}$ for all $j$.

Please observe that, in our ACBC scheme, $\alpha_{\max }$ is achieved when no UE arrivals occur during $M$ consecutive RAOs, which gives $u(j-m)=1$ for $m \in\{0,1, \ldots, M-1\}$. In such case, all the resources are idle, hence, we have $\alpha_{\max }=\mu M$. Building on this, the possible values of $\mu$ are bounded by the inequality

$$
0<\mu \leq \frac{2}{M}
$$

One of the most typical applications of the LMS adaptive algorithm is that of an adaptive line enhancer (ALE). An ALE is a system that may be used to detect a sinusoidal or narrowband information-bearing signal buried in a wideband noise background [36, Chapter 6]. In our ACBC scheme, sudden variations of $u(j)$ represent the wideband noise, in which the narrowband information signal is buried. In other words, $u(j)$ is affected by the randomness of both, the distribution of UE arrivals and of the RA procedure. Hence, the filter weights are automatically adjusted by the LMS algorithm to suppress the sudden variations of $u(j)$.

In this study we propose and evaluate the performance of two different configurations of the LMS ALE. The first one is the typical ALE configuration and the second one, is a novel twist in the ALE configuration that causes the LMS algorithm to pull towards a desired output which is selected empirically. Hereafter we refer to the latter as the pulling adaptive line enhancer (PALE) configuration. The differences between these two configurations are now described in detail.

ALE: This a typical ALE configuration, in which the desired response (primary input) is the ratio of idle to available resources calculated at the $j$ th SIB2 broadcast interval $d(j)=u(j)$. The (reference) input, is a delayed version of the latter. That is, $u(j-\Delta)$, where $\Delta$ is the decorrelation delay. Therefore, the input vector is given as

$\boldsymbol{u}(j-\Delta)=[u(j-\Delta), u(j-\Delta-1), \ldots, u(j-\Delta-M+1)]$.

By implementing the ALE configuration, the filter weights are automatically adjusted to minimize the error between $u(j)$ and its past values $u(j-\Delta-m)$ for $m \in\{0,1, \ldots, M-1\}$. As a consequence, sudden variations are suppressed from $y(j)$. To implement this configuration, it is sufficient to substitute $d(j)$ with $u(j)$, and $\boldsymbol{u}(j)$ with $\boldsymbol{u}(j-\Delta)$ in equations (12), (13a), and (13b) of Algorithm 2. Fig. 4 shows the block diagram of the ALE-LMS.

A consideration of importance in this configuration is that the decorrelation delay $\Delta$ must be sufficiently large so the noise in $u(j)$ is not correlated with that in $u(j-\Delta)$. We have observed that, since $t_{\mathrm{si}}$ is large when compared to $t_{\text {rao }}$, it is sufficient to set $\Delta=1$.

PALE: This is a new twist on the typical ALE configuration, in which the desired response (primary input) $d(j)$ is set to be a constant, selected empirically. On the other hand, the (reference) input is simply $u(j)$. That is,

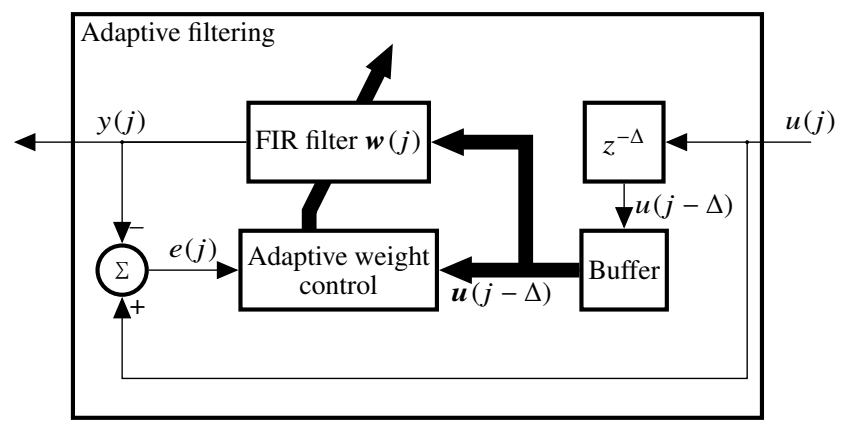

Figure 4: Block diagram of the adaptive line enhancer (ALE) with the LMS adaptive algorithm.

no correlation exists between the constant $d(j)$, and the variations in $u(j-\Delta)$ for all $\Delta \in \mathbb{Z}_{\geq 0}$. Therefore we can set $\Delta=0$.

By implementing the PALE configuration, the filter weights are automatically adjusted to minimize the error between $d(j)$ and $u(j)$, and to suppress the sudden variations of the latter. As a result, $p_{\text {acb }}(j)$ is pulled towards $d(j)$. Building on this, we suggest to set $d(j)=1$. That is, equal to the maximum value of $u(j)$, which is only obtained when all the resources during the SIB2 broadcast interval are idle. As it will be seen in Section 6, setting $d(j)=1$ minimizes the delay of UEs during intervals of low signaling traffic intensity.

\section{Test scenarios, tools, and methodology}

In this section we present the scenarios, the tools, and the methodology that will be used to assess the performance of our ACBC scheme.

Access control schemes must provide an adequate performance under several traffic conditions and network configurations. Hence, we evaluate the performance of the LTE-A RA procedure with our ACBC scheme under the following traffic models.

1. Traffic model 1: An interval in which $n=30000$ UE arrivals follow a uniform distribution within a period of $t_{\text {dist }}=60 \mathrm{~s}$ [10]. This scenario corresponds to a period with a massive number of UE arrivals, still the number of UE arrivals per RAO $a(i)$ is small when compared to the theoretical signaling capacity. Hence, $\approx 100$ percent of the UEs successfully complete the RA procedure even when the ACB scheme is disabled. The performance evaluation under this scenario is relevant because it allows us to determine whether the UE arrivals that occur within intervals of low signaling traffic intensity are affected by the implemented ACBC scheme. For example, with an additional and unnecessary delay.

2. Traffic model 2: A high congestion interval in which $n=30000$ UE arrivals follow a $\operatorname{Beta}(3,4)$ distribution within a distribution period of $t_{\text {dist }}=10 \mathrm{~s}$ 
Table 1: Characteristics of the different traffic models defined by the $3 \mathrm{GPP}$ for the performance evaluation of the RA procedure [10].

\begin{tabular}{lll}
\hline Parameter & Traffic model 1 & Traffic model 2 \\
\hline Number of UEs $n$ & 30000 & 30000 \\
Distribution period $t_{\text {dist }}(\mathrm{s})$ & 60 & 10 \\
Distribution over $t_{\text {dist }}$ & Uniform & Beta $(3,4)$ \\
\hline
\end{tabular}

Table 2: Parameters for the selected network configuration.

\begin{tabular}{ll}
\hline Parameter & Setting \\
\hline Available preambles & $r \in\{30,54\}$ \\
Subframe length & $1 \mathrm{~ms}$ \\
RAO periodicity & $t_{\mathrm{rao}}=5$ subframes \\
RAR window size & 5 subframes \\
Available uplink grants per RAR window & $n_{\mathrm{ug}}=15$ \\
si-Periodicity & $80 \mathrm{~ms}$ \\
SIB2 periodicity in RAOs & $t_{\mathrm{si}}=16$ \\
Maximum number of preamble transmissions & 10 \\
Backoff indicator & $20 \mathrm{~ms}$ \\
Detection probability for the $k$ th preamble & $P_{d}(k)=1-1 / e^{k}$ \\
transmission & 5 \\
Maximum number of $M s g 3$ and $M s g 4$ transmis- & 5 \\
sions & 0.9 \\
Detection probability for $M s g 3$ and $M s g 4$ & \\
\hline
\end{tabular}

[10]. The performance evaluation under this traffic model is the one that has attracted the most attention from the research community [10, 11, 24, 32] because only 31.31 percent of the UEs successfully complete the RA procedure when a typical configuration is selected.

The main characteristics of these two traffic models are shown in Table 1.

The most common configuration for the PRACH is adopted along with other common settings and assumptions regarding the RA procedure $[10,11,24,32]$. The selected configuration parameters for the $\mathrm{RACH}$ and the PDCCH, which are common for both traffic models are shown in Table 2. We assume a similar configuration for the NB-IoT PRACH and PDCCH.

A total of 48 and 64 preambles exist in NB-IoT and in LTE-A respectively. However, the eNB commonly reserves some preambles for high priority UEs in LTE-A and for higher CE levels in NB-IoT. The most typical setting for the number of available preambles for the contention-based RA procedure in LTE-A is $r=54$, in which ten out of the total 64 preambles are reserved for contention-free RA [10, 11, 24, 32]. On the other hand, setting $r=30$ for CE level zero in NB-IoT allows for the reservation of the remaining 18 preambles for UEs with a higher CE level.

These two different values of $r$ are quantitatively different from the perspective of our ACBC scheme. To showcase this difference, let $S_{i}$ be the RV that defines the number of assigned uplink grants at the $i$ th RAO. We show the expected value of $S_{i}$ as a function of $n(i)$ for a given $r \in$

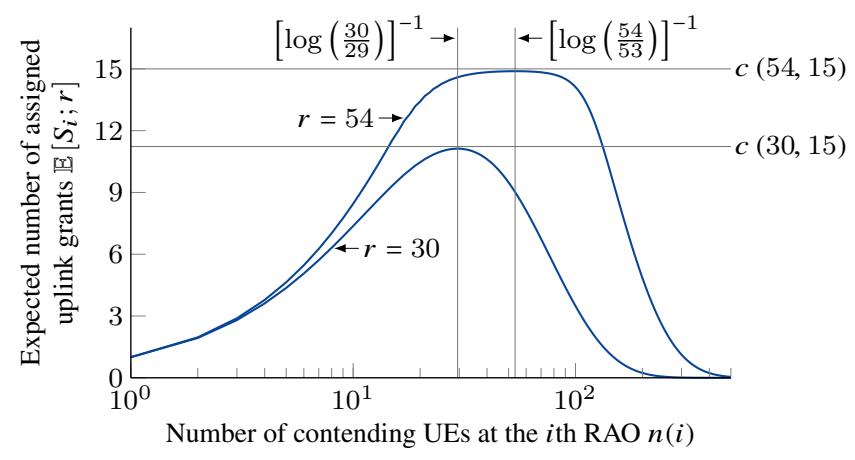

Figure 5: Expected number of assigned uplink grants $\mathbb{E}\left[S_{i} ; r\right]$ as a function of the number of contending UEs at the $i$ th RAO $n(i)$ for $r \in\{30,54\}$ and $n_{\mathrm{ug}}=15$. The $\mathrm{x}$-axis is shown in logarithmic scale.

$\{30,54\}$, denoted as $\mathbb{E}\left[S_{i} ; r\right]$ in Fig. 5 . It can be seen that $\mathbb{E}\left[S_{i} ; r\right]$ is an increasing function that reaches its absolute maximum when the number of preamble transmissions per $\mathrm{RAO}$ is $[\log (r /(r-1))]^{-1}$. Hence, our ACBC scheme will decrease $p_{\text {acb }}(j)$ as $n(i)$ increases until $[\log (r /(r-1))]^{-1}$; this is the desired behavior. Conversely, $\mathbb{E}\left[S_{i} ; r\right]$ becomes a decreasing function for $n(i)>[\log (r /(r-1))]^{-1}$, which in turn causes our ACBC scheme to increases $p_{\text {acb }}(j)$. In other words, the operation of our ACBC scheme is adequate if $n(i) \leq[\log (r /(r-1))]^{-1}$.

It can also be observed in Fig. 5 that the rate of change of $\mathbb{E}\left[S_{i} ; r\right]$ is higher for $r=30$ than for $r=54$, especially as $n(i) \rightarrow[\log (r /(r-1))]^{-1}$. The main reason for this is that $c(30,15)$ (i.e., the theoretical signaling capacity for $r=30)$ is limited by the number of preambles. On the other hand, $c(54,15)$ is mainly limited by $n_{\mathrm{ug}}=15$. As will be confirmed throughout the following section, this in turn makes the adequate configuration of our ACBC scheme more challenging for $r=30$ than for $r=54$.

Results were obtained by means of a C-based simulator that closely replicates the arrival process of the UEs, the ACB scheme, and the RA procedure as described in the specifications $[7,8]$. In each simulation, the adaptive algorithm is initialized as described previously and the filter weights are stabilized. Then, $n=30000 \mathrm{UE}$ arrivals are scheduled within the distribution period, $t_{\text {dist }}$, which begins at $i=0$. The $j$ th SIB2 is broadcast at the $\left(j t_{\mathrm{si}}+i_{r}\right)$ th RAO, where $i_{r}=\mathcal{U}\left[0, t_{\mathrm{si}}-1\right]$ is a discrete random time shift. A simulation run ends when every UE has terminated the RA procedure. The number of simulation runs is set to the smallest number that ensures that all the cumulative KPIs obtained up to the last simulation differ from those obtained up to the previous simulation by less than 0.01 percent.

\subsection{Performance metrics and adequate configuration}

In this paper, we assess the performance in terms of the following KPIs.

1. Success probability $P_{s}$ defined as the probability to successfully complete the RA procedure within the maximum number of preamble transmissions. 
2. Access delay $D$ defined as the time elapsed between the first access attempt (barring check or preamble transmission) of a UE and the successful completion of its RA procedure. It is assessed in terms of the 95th percentile $D_{95}$ given in seconds. That is, the delay of 95 percent of the UEs that successfully complete the RA procedure is $D_{95}$ or less. The performance under the traffic model 1 is assessed in terms of the increase in delay due to the implementation of an ACBC scheme given as $\Delta D_{95}=D_{95}-D_{95}^{*}$, where $D_{95}^{*}$ is the 95 th percentile of access delay obtained with no ACB scheme for the selected configuration.

3. Number of preamble transmissions performed by the UEs that successfully complete the RA procedure $K$. It is assessed in terms of its expected value $\mathbb{E}[K]$.

The methodology for our study is as follows. We first find an adequate value of parameter $\mu$. For this, we observe the behavior of our ACBC scheme under the traffic model 1 for different values of $\mu$ in the range defined by (17). An adequate value of $\mu$ is selected empirically based on the response from the adaptive filter. Specifically, we aim to suppress the sudden variations of $u(j)$ while achieving the fastest possible convergence toward its expected value $\mathbb{E}[u(j)]$. It is important to emphasize that similar trial and error approaches to select $\mu$ are the most common in the practice [39]. The reason for this is that $\mu$ depends on several factors that are application-specific and may not be known. Consequently, the selection of an optimal value is oftentimes impossible.

Then, we continue to find the "optimal" configuration of our ACBC scheme. It is defined as the combination of the barring indicator $t_{\max }$, filter length $M$, and exponent $\omega$ that leads to the minimum $D_{95}$ under both traffic models given $P_{s} \geq 0.95$ under the traffic model 2 for a given $r$. We denote the optimal values of these parameters as $t_{\max }^{*}$, $M^{*}$, and $\omega^{*}$, respectively.

Duan et al. [17] proposed an idealized full state information scheme that is used as a benchmark for their ACBC scheme; the latter is called D-ACB. As described by Duan et al. [17], the benchmark scheme has full state information on the number of contending UEs at each RAO, hence, it can select the optimal barring rate accordingly. On the other hand, their D-ACB scheme estimated the number of contending UEs based on the number of successful and idle preambles, but also on the number of previously registered UEs.

It is important to observe that all of the ACBC schemes presented by Duan et al. [17] were designed for an idealized ACB scheme. That is, they assume the barring parameters are calculated and transmitted at each RAO and also that every UE is subject to the ACB scheme even after initiating the RA procedure. This is not the behavior of the ACB scheme as defined in the protocol specifications [7].

Therefore, we have extended the original benchmark scheme proposed by Duan et al. [17] to cope with the pe- riodicity of the SIB2 $t_{\mathrm{si}}$. As such, the optimal barring rate is calculated as

$$
p_{\mathrm{acb}}^{*}(j)=\min \left\{1, \frac{r}{n^{\prime}(j)}\right\}
$$

where

$$
n^{\prime}(j)=\frac{1}{t_{\mathrm{si}}} \sum_{i=(j-1) t_{\mathrm{si}}+1}^{j t_{\mathrm{si}}} n(i) ;
$$

please recall that $n(i)$ is the number of contending UEs at the $i$ th RAO.

Also, please observe that (19) is exactly as defined by Duan et al. [17] for $t_{\mathrm{si}}=1$, and we simply introduce $n^{\prime}(j)$ to obtain the average optimal barring rate for any $t_{\mathrm{si}} \geq 1$ RAO. Hereafter we refer to this extended scheme simply as the idealized full state information (IFI) scheme; it is used to assess the performance of our ACBC scheme. The barring time $t_{\mathrm{acb}}(j)$ at each barring check under both schemes is deterministic of one RAO [17].

In the following section, we present relevant results derived from the performance analysis of both, the ALE and PALE configurations, along with their optimal parameter configurations.

\section{Results and discussion}

In this section we present and discuss relevant results obtained from the performance evaluation of the RA procedure with our ACBC scheme. As a starting point, we find an adequate value for parameter $\mu$. Then, we compare the performance of our ACBC scheme with that of: 1) our ACBC scheme with no filtering process; 2) a static ACBC scheme with fixed $p_{\text {acb }}^{*}(j)$ and $t_{\text {acb }}^{*}(j)$; and 3$)$ the IFI scheme. The optimal configuration of each of these schemes is assumed. Next, we showcase the robustness of our ACBC scheme by evaluating the impact that deviations from the optimal configuration have on performance. Finally, we discuss the impact of realistic assumptions on the performance of the IFI scheme.

We investigate the impact of $\mu$ on the response of the adaptive algorithm by observing its behavior under the traffic model 1. For this, Fig. 6 shows the response of the algorithm during the first 100 SIB2 transmissions with the ALE configuration for $\mu \in\{2 / M, 1 /(25 M), 1 /(50 M)\}$. Results from a single simulation run are shown to showcase the impact of $\mu$; we have confirmed that these results represent the common behavior of the adaptive algorithm. Typical values $M=32$ and $r=54$ have been selected and UEs ignore the ACB scheme (e.g., were assigned to high priority ACs). That is, at this point we are only interested in observing the difference between the calculated $u(j)$ and $p_{\text {acb }}(j)$, not in their effect in the UE arrivals.

In particular, we are set to find a setting for $\mu$ that successfully reduces the variations of $u(j)$ with the fastest possible convergence toward $\mathbb{E}[u(j)]$. Under the traffic model $1, n=30000$ UE accesses are uniformly distributed within 


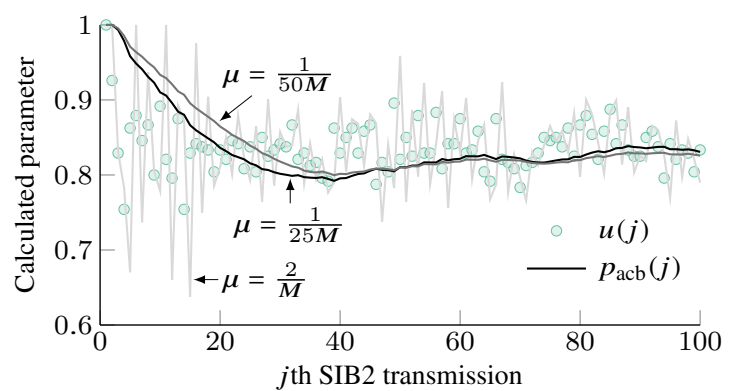

Figure 6: Ratio of idle to available resources $u(j)$ and barring rate $p_{\text {acb }}(j)$ calculated at the $j$ th SIB2 for $\mu \in\{2 / M, 1 /(25 M), 1 /(50 M)\}$; UEs ignore the ACB scheme.

60 s. Next, let $A$ be the RV that defines the number of $\mathrm{UE}$ accesses at an arbitrary RAO within the distribution period. Hence, $a(i)$ is the outcome of a single experiment for RV $A$ at the $i$ th RAO. Given $t_{\text {rao }}=5$ subframes, we have $\mathbb{E}[A]=n / 12000=2.5$. From there, the following approximation can be obtained by substituting $s(i)$ with $\mathbb{E}[A]$ in $(5)$ and $(6)$.

$$
\mathbb{E}[u(j)] \approx 1-\frac{1}{t_{\mathrm{si}} c\left(r, n_{\mathrm{ug}}\right)} \sum_{i=(j-1) t_{\mathrm{si}}+1}^{j t_{\mathrm{si}}} \mathbb{E}[A]=1-\frac{\mathbb{E}[A]}{c\left(r, n_{\mathrm{ug}}\right)}
$$

which gives $\mathbb{E}[u(j)] \approx 5 / 6$ for $r=54$. This value has been confirmed by simulation and by an analytical model [16].

It can be seen in Fig. 6 that the maximum possible value of $\mu=2 / M$ does not provide the desired response because the variations of $p_{\text {acb }}(j)$ are even greater than that of $u(j)$. On the other hand, the LMS algorithm behaves as a low-pass filter with a sharp cutoff bandwidth that successfully suppresses the sudden variations of $u(j)$ when lower values of $\mu$ are selected. However, it can also be observed in Fig. 6 that $\mu=1 /(50 M)$ induces a slightly higher delay than $\mu=1 /(25 M)$. That is, the curve for $\mu=1 /(50 M)$ converges more slowly toward $\mathbb{E}[u(j)]$ than the curve for $\mu=1 /(25 M)$ and the variations of both are comparable. Hence, $\mu=1 /(25 M)$ is used throughout the remainder of the paper. The interested reader is referred to [36, Chapter 6] for more details on the impact of $\mu$ in the response of the LMS algorithm.

\subsection{Performance of $A C B C$ schemes with the optimal con- figuration}

We begin our performance analysis by presenting the optimal configuration of the selected ACBC schemes given $t_{\mathrm{si}}=16$ RAOs in Table 3. As mentioned above, the optimal configuration of each ACBC scheme is defined as the configuration that leads to the shortest $D_{95}$ under both traffic models, given $P_{s} \geq 0.95$ under the traffic model 2. To find the optimal configuration of our ACBC scheme, we have evaluated the performance with $\omega \in \mathbb{Z}_{\geq 0}$, $M \in\{1,2,4, \ldots, 128\}$, and $t_{\max } \in\{0.1,0.2, \ldots, 10\}$ s for each $r \in\{30,54\}$. We have observed that the optimal value of
Table 3: Optimal configuration of the different ACBC schemes.

\begin{tabular}{llll}
\hline \multirow{2}{*}{ ACBC scheme } & Parameter & \multicolumn{2}{c}{ Optimal value } \\
\cline { 3 - 4 } & & $r=30$ & $r=54$ \\
\hline ALE & $\omega$ & 3 & 3 \\
& $M$ & 32 & 32 \\
PALE & $t_{\max }$ & 3.8 & 0.3 \\
\multirow{2}{*}{ No filtering } & $M$ & 16 & 32 \\
& $t_{\mathrm{acb}}(j)=t_{\max }$ & 4.2 & 0.6 \\
Static & $\omega$ & 0 & 2 \\
& $t_{\max }$ & 5.4 & 3.3 \\
& $p_{\mathrm{acb}}(j)$ & 0.11 & 0.31 \\
& $t_{\mathrm{acb}}(j)$ & 1.2 & 1.75 \\
\hline
\end{tabular}

the mean barring time for the PALE configuration is simply $p_{\mathrm{acb}}^{*}(j)=t_{\max }^{*}$.

The KPIs obtained under both traffic models with the optimal configuration of each of the selected ACBC schemes are presented in Table 4 . KPIs obtained with no implemented ACB scheme have been included as a reference. The success probability $P_{s}$ under the traffic model 1 has been omitted because it is equal to one for all cases.

It is important to emphasize that the IFI scheme cannot be implemented in current cellular systems. As a consequence, the performance reported in Table 4 for the IFI scheme is not achievable in practice. However, it provides an upper bound for the performance of the ACB scheme. A detailed study on the impact of realistic assumptions on the performance of the IFI scheme is presented on page 13 .

Table 4 reveals that $P_{s} \geq 0.95$ can be obtained with any of the selected ACBC schemes under the traffic model 2 for both $r \in\{30,54\}$. As it can be seen, the $D_{95}$ obtained with our ACBC scheme with the ALE and PALE configurations is up to 50 percent shorter than with no filtering process. This showcases the benefits the adaptive filter. Moreover, the $D_{95}$ obtained with our ACBC under the traffic model 2 is, in the worst case, around 28 percent longer than the one obtained with the IFI scheme. This difference is significant, but is important to emphasize that our ACBC scheme can be implemented at the eNBs in its current form.

Yet another interesting aspect is that the $D_{95}$ obtained with our ACBC scheme under the traffic model 2 is around 48 percent shorter than the one obtained with the static ACBC despite the long period $t_{\mathrm{si}}=16$. Still, the achieved $D_{95}$ with any of the ACBC schemes under the traffic model 2 is in the order of a few seconds; such long delay is only suitable for delay-tolerant applications.

Needless to say, the optimal performance under the traffic model 1 is obtained with no ACB scheme, but also with the IFI scheme. That is, the effect of the deterministic barring time of one RAO combined with a sufficiently high $p_{\text {acb }}^{*}(j)$ is not observable in the selected KPIs. On the other hand, the longest $D_{95}$ under the traffic model 2 is obtained with the static ACBC and a similar $D_{95}$ is obtained under the traffic model 1. Naturally, the static ACBC is not an 
Table 4: KPIs obtained with the optimal configuration of the selected ACBC schemes and with no ACB scheme under both traffic models.

\begin{tabular}{|c|c|c|c|c|c|c|c|c|c|c|}
\hline \multirow[b]{3}{*}{ ACBC scheme } & \multicolumn{4}{|c|}{ Traffic model 1} & \multicolumn{6}{|c|}{ Traffic model 2} \\
\hline & \multicolumn{2}{|c|}{$\begin{array}{c}\text { 95th percentile } \\
\text { of access } \\
\text { delay (s) }\end{array}$} & \multicolumn{2}{|c|}{$\begin{array}{c}\text { Expected number } \\
\text { of preamble } \\
\text { transmissions }\end{array}$} & \multicolumn{2}{|c|}{$\begin{array}{c}\text { Success } \\
\text { probability }\end{array}$} & \multicolumn{2}{|c|}{$\begin{array}{c}\text { 95th percentile } \\
\text { of access } \\
\text { delay (s) }\end{array}$} & \multicolumn{2}{|c|}{$\begin{array}{c}\text { Expected number } \\
\text { of preamble } \\
\text { transmissions }\end{array}$} \\
\hline & $r=30$ & $r=54$ & $r=30$ & $r=54$ & $r=30$ & $r=54$ & $r=30$ & $r=54$ & $r=30$ & $r=54$ \\
\hline ALE & 0.110 & 0.057 & 1.576 & 1.500 & 0.951 & 0.965 & 14.450 & 6.807 & 2.438 & 2.584 \\
\hline PALE & 0.065 & 0.059 & 1.575 & 1.500 & 0.968 & 0.979 & 14.425 & 7.286 & 2.557 & 2.485 \\
\hline No filtering & 6.984 & 0.165 & 1.567 & 1.500 & 0.997 & 0.967 & 21.440 & 10.839 & 2.065 & 2.189 \\
\hline Static & 30.349 & 13.379 & 1.548 & 1.494 & 0.951 & 0.950 & 30.927 & 13.584 & 2.348 & 2.635 \\
\hline IFI & 0.060 & 0.055 & 1.576 & 1.500 & 0.988 & 0.971 & 11.491 & 5.468 & 3.123 & 3.392 \\
\hline No ACB & 0.060 & 0.055 & 1.575 & 1.500 & 0.115 & 0.313 & 0.175 & 0.182 & 3.157 & 3.452 \\
\hline
\end{tabular}

efficient solution to congestion.

Table 4 also shows that the $D_{95}$ obtained with our ACBC scheme under the traffic model 1 is less than seven percent higher than the minimum, achieved with no ACB scheme. The only exception occurs with the ALE configuration for $r=30$. In this case, setting $\omega=3$ is not sufficient to achieve a lower $D_{95}$ and, as it will be discussed later in this section, selecting $\omega \geq 4$ sharply increases $t_{\max }^{*}$ to the point that there is no $t_{\max } \leq 10 \mathrm{~s}$ that leads to $P_{s} \geq 0.95$ under the traffic model 2. Moreover, the effect of increasing $\omega$ on $t_{\max }^{*}$ is magnified if no filtering process is incorporated to our ACBC scheme. Concretely, no combination of $t_{\max }$ and $\omega>0$ given $r=30$ exists for which $P_{s} \geq 0.95$ and selecting $\omega=0$ results in an excessively long access delay under the traffic model 1 .

We have also evaluated the performance of our ACBC scheme with the optimal configuration shown in Table 3 under congestion scenarios comparable to the traffic model 2. For instance, when $n=30000 \mathrm{UE}$ arrivals follow a Beta $(4,4)$ distribution over $t_{\text {dist }}=10 \mathrm{~s}$. The peak in the average number of UE arrivals is around five percent higher for Beta $(4,4)$ than for Beta $(3,4)$. The performance of our ACBC scheme under this traffic model is comparable to that under the traffic model 2 (see Table 4) as $P_{s} \geq 0.93$ is achieved with both the ALE and PALE configurations for $r \in\{30,54\}$. Furthermore, the difference in $D_{95}$ between these two traffic models is less than one percent.

Now we proceed to compare the behavior of the ALE and PALE configurations. For this, Fig. 7 shows the ratio of idle to available resources $u(j)$ and $p_{\text {acb }}(j)$ with the optimal ALE and PALE configurations given $r=54$. A similar behavior was observed for $r=30$, so these results have been omitted.

It is important to point out that the first $12000 \mathrm{RAOs}$ after the beginning of the distribution period are shown in Fig. $7 \mathrm{a}$ and Fig. $7 \mathrm{~b}$ as $t_{\mathrm{si}}=16$ RAOs. On the other hand, the first 3200 RAOs are shown in Fig. 7c and Fig. 7d. Again, results from a single simulation run are shown and we have confirmed that these correspond to the common behavior of our ACBC scheme.

We can clearly observe in Fig. 7 that the filtering pro- cess smooths out the sudden variations (noise) of $u(j)$. The result is a much more stable and accurate selection of $p_{\text {acb }}(j)$. Also it can be seen that the calculated $u(j)$ with the ALE configuration under the traffic model 1 (see Fig. 7a) is similar to the one calculated with the PALE configuration (see Fig. 7b) despite the fact that, for the former, $p_{\text {acb }}(j)<1$ for all $j$. This is caused by the selection of $\omega^{*}=3$ and $t_{\text {max }}^{*}=0.3 \mathrm{~s}$, which results in $t_{\mathrm{acb}}(j) \approx 1 \cdot 10^{-3} \mathrm{~s}$ for all $j$, which is negligible.

On the other hand, the "pulling" effect of the PALE configuration can be clearly observed in Fig. $7 \mathrm{~b}$ and in Fig. 7 d. That is, $p_{\text {acb }}(j)>u(j)$ for most $j$ with the PALE configuration under the traffic model 2 , and for every $j$ under the traffic model 1 . This effect is emphasized by the red arrows, which indicate the difference in amplitude between $u(j)$ and $p_{\text {acb }}(j)$. For instance, Fig. 7c clearly shows that $u(20)<p_{\text {acb }}(20)$, while $u(160)>p_{\text {acb }}(160)$ with the ALE configuration. This is caused by the delay in the response of the algorithm. On the other hand, Fig. $7 \mathrm{~d}$ shows that $u(j)<p_{\text {acb }}(j)$ for both $j \in\{20,160\}$ with the PALE configuration. Although the difference between $u(160)$ and $p_{\text {acb }}(160)$ is considerably small.

\subsection{Robustness of the proposed $A C B C$ scheme}

In this subsection we showcase the robustness of our ACBC scheme by showing the impact that deviations from the optimal ALE and PALE configurations have on performance.

We first investigate the impact of deviations from the optimal value of $\omega$ on the performance of the ALE configuration. For this, the obtained $\Delta D_{95}$ (under the traffic model 1 ) is shown in Fig. 8 a and the obtained $D_{95}$ under the traffic model 2 is shown in Fig. 8 b for $\omega \in\{0,1, \ldots, 7\}$ and $r \in\{30,54\}$, given $t_{\max }^{*}$. Fig. 8 only shows plots corresponding to $r=30$ when $\omega \leq 3$ because there is no $t_{\max }^{*} \leq 10 \mathrm{~s}$ for $\omega>4$. That is, there is no $t_{\max } \leq 10 \mathrm{~s}$ that leads to $P_{s} \geq 0.95$ for $\omega \geq 4$ when $r=30$. This same occurs for $\omega \geq 8$ when $r=54$.

Fig. 8a also shows that high values of $\omega$ sharply decrease $\Delta D_{95}$ but, as described above, excessively high values of $\omega$ may greatly increase $t_{\max }^{*}$. Building on this, $\omega$ 


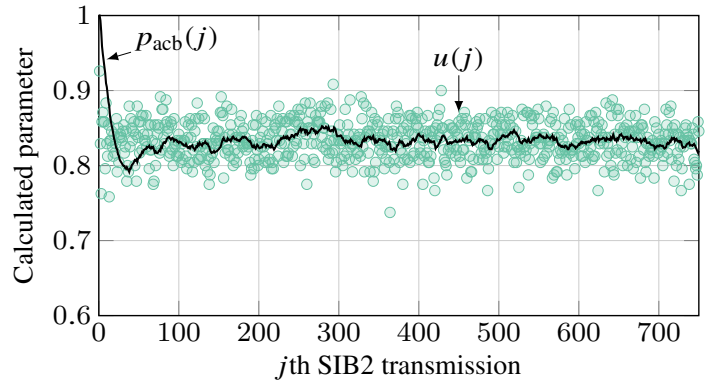

(a)

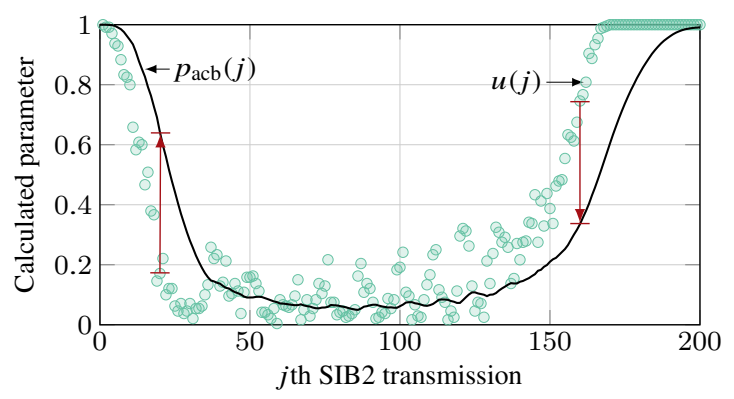

(c)

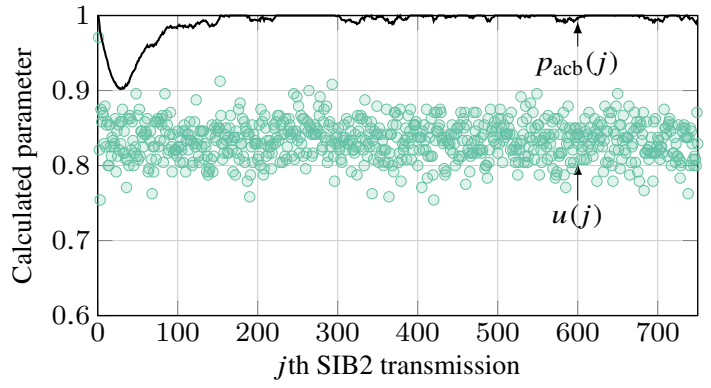

(b)

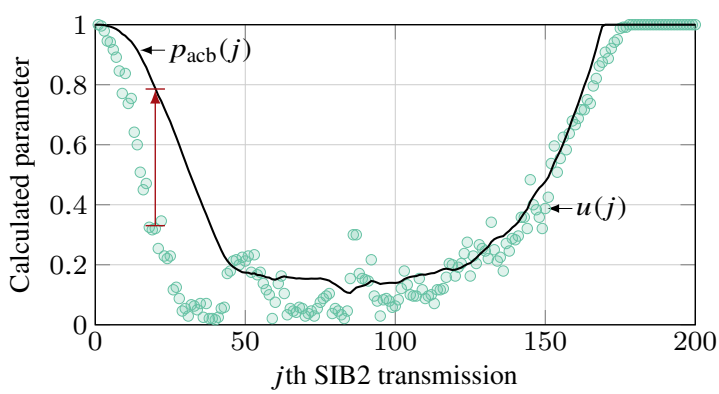

(d)

Figure 7: Ratio of idle to available resources $u(j)$ and barring rate $p_{\text {acb }}(j)$ calculated at the $j$ th SIB2 for a single simulation run and $r=54$ for the cases: (a) ALE, traffic model 1; (b) PALE, traffic model 1; (c) ALE, traffic model 2; and (d) PALE traffic model 2.

must be selected to reduce the access delay under the traffic model 1, but also to achieve an adequate response under the traffic model 2, especially if $r=30$.

Next, we evaluate the impact on performance of deviations from $M^{*}$ and from $t_{\max }^{*}$, given that $\omega^{*}$ is selected. For this, we illustrate $P_{S}$ and $D_{95}$ under the traffic model 2 for the ALE and PALE configurations in Fig. 9; $M \in\{8,16,32,64\}$ and $t_{\max } \in\{0.1,0.2, \ldots, 5\}$ s. Again, only results for $r=54$ are shown as a similar behavior was observed for $r=30$. Results obtained with no ACB scheme are also included as a reference.

Fig. 9 shows that the $P_{s}$ obtained with our ACBC scheme is higher than that with no ACB with any $t_{\max } \in$ $\mathbb{R}_{>0}$. It can also be observed that $P_{s}>0.95$ for all $t_{\max }>t_{\max }^{*}$. That is, selecting $t_{\max }>t_{\max }^{*}$ results in an adequate $P_{S}$ but slightly increases $D_{95}$. For example, $D_{95}=8.380$ and $D_{95}=8.108$ for the ALE and PALE configurations respectively if an intuitive value $t_{\max }=1 \mathrm{~s}$ is selected along with $M^{*}=32$. On the other hand, selecting $t_{\max }<t_{\max }^{*}$ results in a drastic drop in $P_{s}$, except for the ALE configuration with $M=16$. Building on this, and on the fact that in a real world implementation it would be hard to select $t_{\max }^{*}$ since the exact distribution of the arrivals is ignored, it is advisable to follow a preventive approach and select a relatively high $t_{\max }$.

Also, it can also be observed from Fig. 9 that the best performance is obtained with $M=32$. That is, the lowest $t_{\max }^{*}$ was obtained by selecting $M=32$, which leads to the lowest $D_{95}$. However, the performance obtained with other values of $M$ is just slightly inferior. Consequently, the performance of our ACBC scheme is not greatly affected by the selected value of $M$, given that excessively short or long values are avoided.

\subsection{Impact of realistic assumptions on the performance of the IFI scheme}

We conclude our performance analysis by evaluating the performance of the IFI scheme under different scenarios. By doing so, we illustrate the impact of the factors that hinder the accurate selection of barring parameters. We assume the eNB always has perfect information on the number of UEs that will perform an access attempt at each RAO (i.e., even before the RAO occurs), and hence, can select the optimal barring rate as in (19).

The scenarios are defined by two different factors. The first one is the selectivity of the ACB scheme and the second one is the periodicity of the SIB2 $t_{\mathrm{si}}$. That is, we assume that either: 1) every UE is subject to the ACB scheme; or 2) only the UEs that have not yet begun the RA procedure are subject to the ACB scheme. We also consider $t_{\mathrm{si}} \in\{1,16\}$. Hence, we consider the hypothetical case in which $t_{\mathrm{si}}=1$ besides the lowest achievable $t_{\mathrm{si}}=16$ RAOs in LTE-A. The combination of these two factors results in the four scenarios included in Table 5, where we show the $P_{s}$ obtained with the IFI scheme.

As it can be seen, the main factor that hinders the proper operation of the IFI scheme is the selectivity of the ACB scheme. That is, the IFI scheme can deal with the real periodicity of the SIB2 because a sufficiently high $P_{S} \geq 0.95$ is achieved when every UE is subject to the 


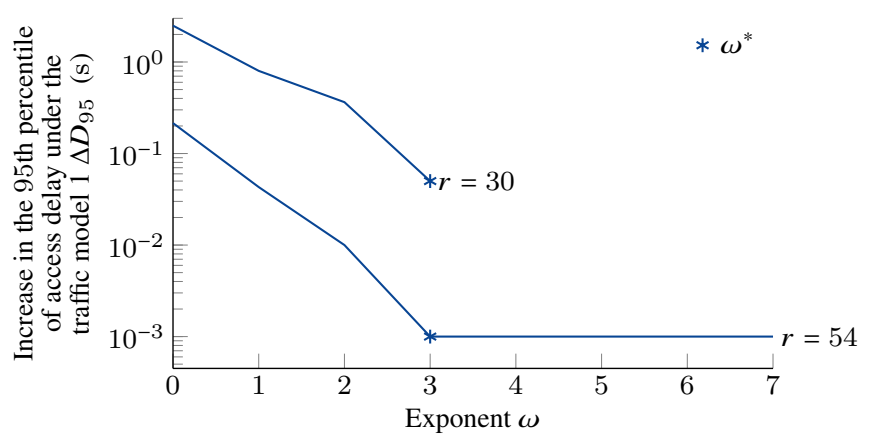

(a)

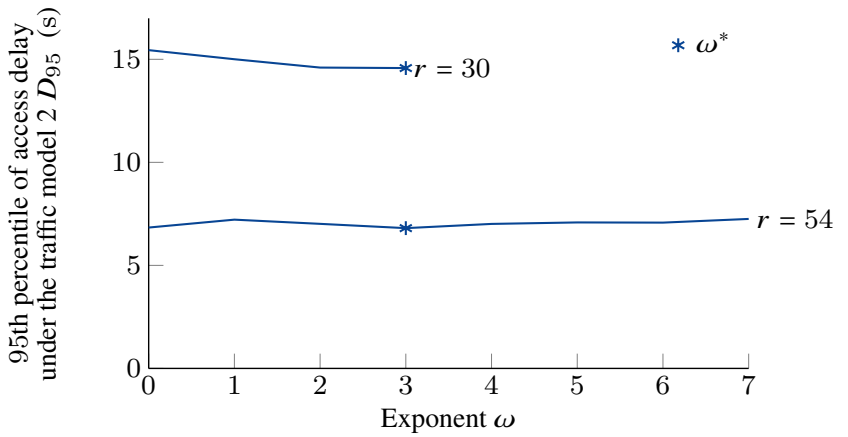

(b)

Figure 8: (a) Increase in the 95 th percentile of access delay under the traffic model $1 \Delta D_{95}$ and (b) 95 th percentile of access delay $D_{95}$ under the traffic model 2 given $t_{\max }^{*}, M^{*}$, and $\omega$ for the ALE configuration; $r \in\{30,54\}$. No $t_{\max }^{*} \leq 10 \mathrm{~s}$ exists for $\omega \geq 4$ and $r=30$

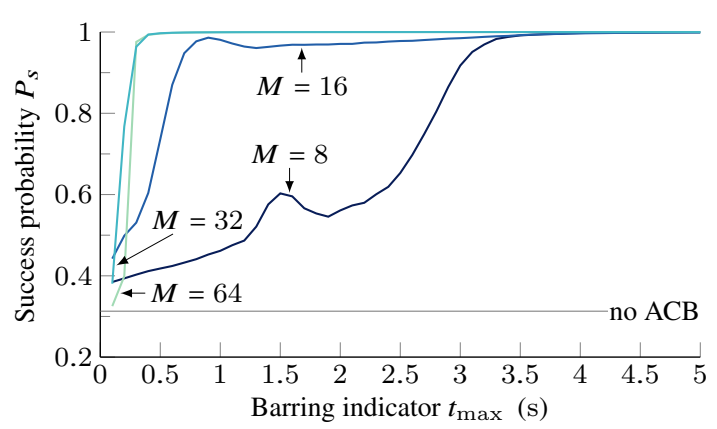

(a)

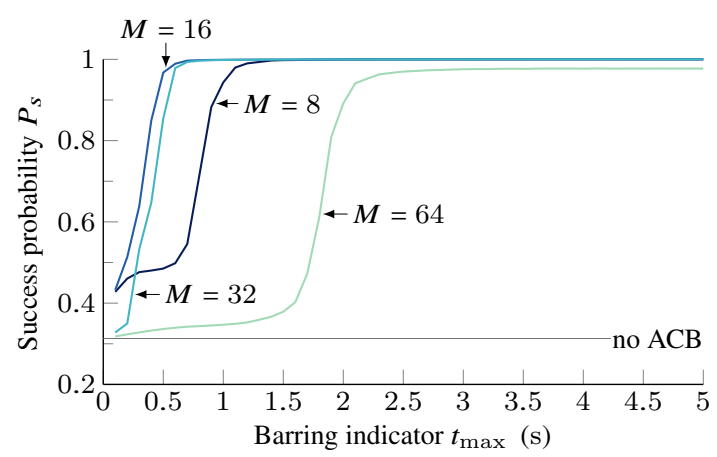

(c)

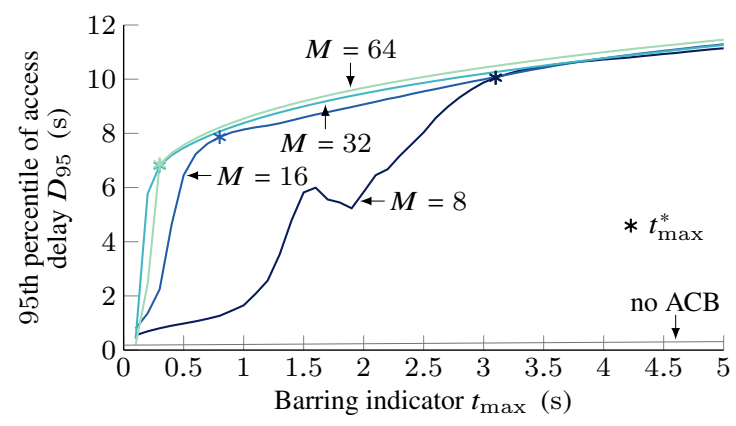

(b)

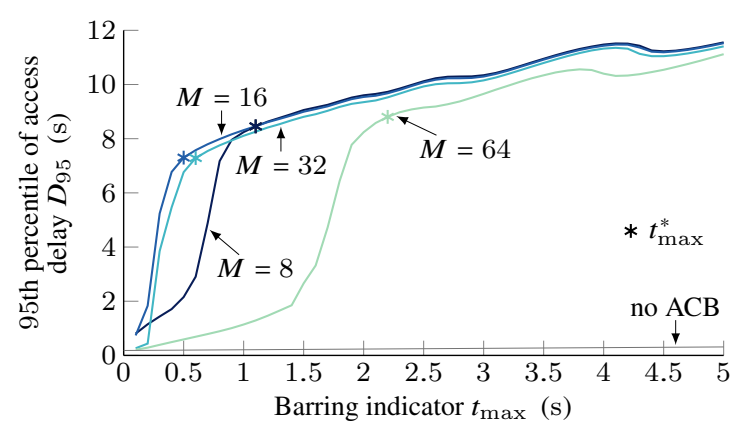

(d)

Figure 9: Success probability $\boldsymbol{P}_{\boldsymbol{S}}$ for the: (a) ALE and (b) PALE configurations, and 95th percentile of access delay $\boldsymbol{D}_{95}$ for the: (c) ALE and (d) PALE configurations as a function of $t_{\max }$ under the traffic model $2 ; r=54$ and $\omega^{*}$.

ACB scheme. On the other hand, the performance of the IFI scheme is poor if the UEs are only subject to the ACB scheme before they initiate the RA procedure.

Concretely, if we compare the IFI scheme in this latter scenario with the case in which no ACB scheme is implemented (see Table 4 on page 12), a similar $P_{s}=0.313$ is obtained with $r=54$. On the other hand, the $P_{s}$ obtained with $r=30$ is even lower with the IFI scheme than with no ACB scheme (i.e., $P_{s}=0.115$ with no ACB scheme). This problem may be solved by an adequate selection of the barring time $t_{\mathrm{acb}}(j)$, but no strategies to fine-tune this parameter were investigated in [17].
Table 5: Success probability obtained with the IFI scheme under different scenarios.

\begin{tabular}{lccc}
\hline \multicolumn{1}{c}{$\begin{array}{c}\text { UEs subject to the } \\
\text { IFI scheme }\end{array}$} & $\begin{array}{c}\text { SIB2 periodicity } \\
t_{\mathrm{si}}\end{array}$ & \multicolumn{2}{c}{$\begin{array}{c}\text { Success } \\
\text { probability }\end{array}$} \\
\cline { 3 - 4 } & & $P_{s}$ \\
\hline Every UE & 1 & 0.987 & 0.988 \\
& 16 & 0.988 & 0.971 \\
Only the UEs that have not & 1 & 0.106 & 0.313 \\
yet begun the RA procedure & 16 & 0.100 & 0.312 \\
\hline
\end{tabular}




\section{Conclusions}

In this paper, we presented a novel ACBC scheme in which the selection of the barring parameters is based on the ratio of idle to available resources. The latter serves as the input to an adaptive filtering process, where the LMS algorithm is implemented. Two different configurations of this algorithm were evaluated: a typical adaptive line enhancer (ALE) and a novel "pulling" adaptive line enhancer (PALE).

Our performance analysis has revealed that the target success probability of 95 percent under the traffic model 2 can be obtained by means of numerous ACBC schemes. Nevertheless, our ACBC scheme is one of the few that combines the following three characteristics: 1) leads to a nearly optimal performance under periods of no congestion; 2) successfully relieves congestion under mMTC scenarios while obtaining a short access delay; and 3) can be directly implemented in current cellular networks. And, to the best of our knowledge, is the one that provides the best performance.

Between the ALE and PALE configurations, the latter is the only configuration that minimizes the access delay during intervals of low signaling traffic intensity for $r=30$. That is, when the signaling capacity is exclusively limited by the number of available preambles. On the other hand, a similar performance can be obtained with both configurations for $r=54$. That is, when the signaling capacity is limited by the number of available uplink grants. The main practical difference between these configurations relies on the ease of correctly setting the configuration parameters. Concretely, the range of adequate values of $t_{\max }$ is larger for the ALE configuration than for the PALE configuration. On the other hand, no exponential $\omega$ is needed for the PALE configuration.

\section{Acknowledgment}

Funding: This research has been supported in part by the Ministry of Economy and Competitiveness of Spain under Grant TIN2013-47272-C2-1-R and Grant TEC201571932-REDT. The research of I. Leyva-Mayorga was partially funded by grant 383936 CONACYT-GEM 2014.

\section{References}

[1] Service requirements for machine-type communications, 3GPP TS 22.368 V13.2.0 (Dec. 2016).

[2] P. K. Verma, R. Verma, A. Prakash, A. Agrawal, K. Naik, R. Tripathi, T. Khalifa, M. Alsabaan, T. Abdelkader, A. Abogharaf, Machine-to-machine (M2M) communications: A survey, J. Netw. Comput. Appl. 66 (2016) 83-105. doi: 10.1016/j.jnca.2016.02.016.

[3] Cisco visual networking index (VNI): Global mobile data traffic forecast update, 2016-2021 white paper, accessed: Aug. 12, 2018 (Feb. 2017).

URL http://www.cisco.com/c/en/us/solutions/collateral/ service-provider/visual-networking-index-vni/ mobile-white-paper-c11-520862.html
[4] IMT vision - Framework and overall objectives of the future development of IMT for 2020 and beyond, ITU-R Rec. M.2083 (2015).

[5] F. Ghavimi, H.-H. Chen, M2M communications in 3GPP LTE/LTE-A networks: Architectures, service requirements, challenges, and applications, IEEE Commun. Surveys Tuts. 17 (2) (2015) 525-549. doi:10.1109/COMST.2014.2361626.

[6] Study on scenarios and requirements for next generation access technologies, 3GPP TR 38.913 V14.2.0 (Apr. 2017).

[7] Radio resource control (RRC); Protocol specification, 3GPP TS 36.331 V15.3.0 (Sep. 2018).

[8] Service accessibility, 3GPP TS 22.011 V13.6.0 (Jul. 2016).

[9] Physical channels and modulation, 3GPP TS 36.211 V12.6.0 (Sep. 2015).

[10] Study on RAN improvements for machine-type communications, 3GPP TR 37.868 (Jul. 2011).

[11] C. H. Wei, G. Bianchi, R. G. Cheng, Modeling and analysis of random access channels with bursty arrivals in OFDMA wireless networks, IEEE Trans. Wireless Commun. 14 (4) (2015) 19401953. doi:10.1109/TWC.2014.2377121.

[12] A. Laya, L. Alonso, J. Alonso-Zarate, Is the random access channel of LTE and LTE-A suitable for M2M communications? A survey of alternatives, IEEE Commun. Surveys Tuts. 16 (1) (2014) 4-16. doi:10.1109/SURV.2013.111313.00244.

[13] A. Biral, M. Centenaro, A. Zanella, L. Vangelista, M. Zorzi, The challenges of M2M massive access in wireless cellular networks, Digit. Commun. Networks 1 (1) (2015) 1-19.

[14] E. Soltanmohammadi, K. Ghavami, M. Naraghi-Pour, A survey of traffic issues in machine-to-machine communications over LTE, IEEE Internet Things J. 3 (6) (2016) 865-884. doi: 10.1109/JIOT.2016.2533541.

[15] Y.-P. E. Wang, X. Lin, A. Adhikary, A. Grovlen, Y. Sui, Y. Blankenship, J. Bergman, H. S. Razaghi, A primer on 3GPP narrowband Internet of Things, IEEE Commun. Mag. 55 (3) (2017) 117-123. doi:10.1109/MCOM. 2017.1600510CM.

[16] I. Leyva-Mayorga, L. Tello-Oquendo, V. Pla, J. MartinezBauset, V. Casares-Giner, On the accurate performance evaluation of the LTE-A random access procedure and the access class barring scheme, IEEE Trans. Wireless Commun. 16 (12) (2017) 7785-7799. doi:10.1109/TWC.2017.2753784.

[17] S. Duan, V. Shah-Mansouri, Z. Wang, V. W. S. Wong, D-ACB: Adaptive congestion control algorithm for bursty M2M traffic in LTE networks, IEEE Trans. Veh. Technol. 65 (12) (2016) 9847-9861. doi:10.1109/TVT.2016.2527601.

[18] L. Ferdouse, A. Anpalagan, S. Misra, Congestion and overload control techniques in massive M2M systems: A survey, Trans. Emerg. Telecommun. Technol. 28 (2) (2015) e2936. doi:10. 1002/ett. 2936.

[19] R. Abbas, M. Shirvanimoghaddam, Y. Li, B. Vucetic, Random access for M2M communications with QoS guarantees, IEEE Trans. Commun. 65 (7) (2017) 2889-2903. doi:10.1109/TCOMM. 2017.2690900.

[20] L. Tello-Oquendo, I. Leyva-Mayorga, V. Pla, J. MartinezBauset, J. R. Vidal, V. Casares-Giner, L. Guijarro, Performance analysis and optimal access class barring parameter configuration in LTE-A networks with massive M2M traffic, IEEE Trans. Veh. Technol. 67 (4) (2018) 3505-3520. doi: 10.1109/TVT. 2017.2776868.

[21] C. Kalalas, J. Alonso-Zarate, Reliability analysis of the random access channel of LTE with access class barring for smart grid monitoring traffic, in: Proc. IEEE Int. Conf. Commun. (ICC) Workshops, 2017, pp. 724-730. doi:10.1109/ICCW. 2017. 7962744.

[22] I. Leyva-Mayorga, L. Tello-Oquendo, V. Pla, J. MartinezBauset, V. Casares-Giner, Performance analysis of access class barring for handling massive M2M traffic in LTE-A networks, in: Proc. IEEE Int. Conf. Commun. (ICC), 2016, pp. 1-6. doi:10.1109/ICC. 2016.7510814.

[23] M. Tavana, A. Rahmati, V. Shah-Mansouri, Congestion control with adaptive access class barring for LTE M2M overload using Kalman filters, Comput. Netw. 141 (2018) 222-233. doi:10. 1016/j.comnet.2018.01.044. 
[24] T. M. Lin, C. H. Lee, J. P. Cheng, W. T. Chen, PRADA: Prioritized random access with dynamic access barring for MTC in 3GPP LTE-A networks, IEEE Trans. Veh. Technol. 63 (5) (2014) 2467-2472. doi:10.1109/TVT.2013.2290128.

[25] T. P. C. de Andrade, C. A. Astudillo, L. R. Sekijima, N. L. S da Fonseca, The random access procedure in Long Term Evolution networks for the Internet of Things, IEEE Commun. Mag. 55 (3) (2017) 124-131. doi:10.1109/MCOM.2017.1600555CM.

[26] I. Leyva-Mayorga, M. A. Rodriguez-Hernandez, V. Pla, J. Martinez-Bauset, L. Tello-Oquendo, An adaptive access class barring scheme for handling massive M2M communications in LTE-A, in: Proc. Eur. Wireless Conf., 2017, pp. 143-148.

[27] Z. Wang, V. W. S. Wong, Optimal access class barring for stationary machine type communication devices with timing advance information, IEEE Trans. Wireless Commun. 14 (10) (2015) 5374-5387. doi:10.1109/TWC.2015.2437872.

[28] H. Jin, W. T. Toor, B. C. Jung, J.-B. Seo, Recursive pseudobayesian access class barring for M2M communications in LTE systems, IEEE Trans. Veh. Technol. 66 (9) (2017) 8595-8599. doi:10.1109/TVT .2017.2681206

[29] L. Tello-Oquendo, D. Pacheco-Paramo, V. Pla, J. MartinezBauset, Reinforcement learning-based ACB in LTE-A networks for handling massive $\mathrm{M} 2 \mathrm{M}$ and $\mathrm{H} 2 \mathrm{H}$ communications, in: IEEE Int. Conf. Commun. (ICC), Vol. 2018-May, 2018, pp. 1-7. doi : 10.1109/ICC. 2018.8422167.

[30] O. Arouk, A. Ksentini, General model for RACH procedure performance analysis, IEEE Commun. Lett. 20 (2) (2016) 372375. doi:10.1109/LCOMM. 2015.2505280.

[31] Z. Zhang, H. Chao, W. Wang, X. Li, Performance analysis and UE-side improvement of extended access barring for machine type communications in LTE, in: Proc. IEEE Veh. Technol.
Conf. (VTC Spring), 2014, pp. 1-5. doi:10.1109/VTCSpring. 2014.7023042.

[32] R. G. Cheng, J. Chen, D. W. Chen, C. H. Wei, Modeling and analysis of an extended access barring algorithm for machinetype communications in LTE-A networks, IEEE Trans. Wireless Commun. 14 (6) (2015) 2956-2968. doi:10.1109/TWC.2015. 2398858

[33] Physical layer procedures, 3GPP TS 36.213 V14.2.0 (Apr. 2017).

[34] R. Harwahyu, R.-G. Cheng, C.-H. Wei, Investigating the performance of the random access channel in NB-IoT, in: Proc. IEEE Veh. Technol. Conf. (VTC Fall), 2017, pp. 1-5. doi: 10.1109/VTCFall.2017.8288195.

[35] N. Jiang, Y. Deng, M. Condoluci, W. Guo, A. Nallanathan, M. Dohler, RACH preamble repetition in NB-IoT network, IEEE Commun. Lett. 22 (6) (2018) 1244-1247. doi:10.1109/ LCOMM . 2018. 2793274.

[36] S. Haykin, Adaptive filter theory, 4th Edition, Prentice Hall, NJ, USA, 2002

[37] A. Uncini, Fundamentals of adaptive signal processing, Signals and Communication Technology, Springer, Cham, Switzerland, 2015. doi:10.1007/978-3-319-02807-1.

[38] B. Widrow, J. M. McCool, M. G. Larimore, C. R. Johnson, Stationary and nonstationary learning characteristics of the LMS adaptive filter, Proc. IEEE 64 (8) (1976) 1151-1162. doi:10.1109/PROC.1976.10286.

[39] B. Widrow, J. R. Glover, J. M. McCool, J. Kaunitz, C. S. Williams, R. H. Hearn, J. R. Zeidler, J. E. Dong, R. C. Goodlin, Adaptive noise cancelling: Principles and applications, Proc. IEEE 63 (12) (1975) 1692-1716. doi:10.1109/PROC. 1975. 10036. 